Discussion Paper Series A No.517

\title{
Bargaining Theory over Opportunity Assignments and the Egalitarian Solution
}

\section{Yongsheng $\mathrm{Xu}$}

(Department of Economics, Andrew Young School of Policy Studies

Georgia State University and

China Academy of Public Finance and Public Policy

Central University of Finance and Economics)

and

Naoki Yoshihara

(Institute of Economic Research, Hitotsubashi University)

April, 2009

Institute of Economic Research

Hitotsubashi University

Kunitachi, Tokyo, 186-8603 Japan 


\title{
Bargaining theory over opportunity assignments and the egalitarian solution ${ }^{*}$
}

\author{
Yongsheng $\mathrm{Xu}$ \\ Department of Economics \\ Andrew Young School of Policy Studies \\ Georgia State University, Atlanta, GA 30303, U.S.A. \\ Email: yxu3@gsu.edu \\ and
}

China Academy of Public Finance and Public Policy

Central University of Finance and Economics

Beijing, China

\author{
Naoki Yoshihara \\ Institute of Economic Research \\ Hitotsubashi University \\ 2-4 Naka, Kunitachi, Tokyo, Japan 186-8603 \\ Email: yosihara@ier.hit-u.ac.jp
}

First Version: December, 2005; This version: March 2009

\footnotetext{
*Financial supports from the Department of Economics, Andrew Young School of Policy Studies, Georgia State University, and from the Ministry of Education, Culture, Sports, Science and Technology of Japan are gratefully acknowledged. We also thank Atsushi Kajii, Chiaki Hara, Shinsuke Nakamura, and audiences of the 8th international meeting of the Society for Social Choice and Welfare held at Istanbul in July 2006, Keio University, Kyoto University, and the 12th Decentralization Conference in Japan held at Osaka Prefecture University in October 2006 where this paper was presented for their valuable comments.
} 


\begin{abstract}
This paper discusses issues of axiomatic bargaining problems over opportunity assignments. The fair arbitrator uses the principle of "equal opportunity" for all players to make the recommendation on resource allocations. A framework in such a context is developed and the egalitarian solution to standard bargaining problems is reformulated and axiomatically characterized.
\end{abstract}

JEL Classification Numbers: C71, C78, D60, D63, D70

Keywords: Opportunity sets, bargaining over opportunity assignments, egalitarian solution 


\section{Introduction}

In standard axiomatic bargaining models originated from Nash (1950), a typical interpretation of the solution to bargaining problems is the recommendation made by a "fair arbitrator" such as the Judge in civil trials, or the function of the Dispute Settlement Body in the WTO mechanism, etc. In such models, this recommendation is based solely on players' utilities. In many contexts, however, the "fair arbitrator" may have other principles in mind when making a recommendation.

For instance, consider the distribution issue of a father's inheritance among his children. The father, as a "fair arbitrator," may have the principle of "equal opportunities" for his children and would like to distribute his wealth among his children giving them equal opportunities to do well in their respective lives. Likewise, when educational resources are to be allocated among local public schools, the local government's board of education, as the "fair arbitrator," may propose an allocation that "equalizes" school children's opportunity sets for future jobs, skills, college admissions, lives, etc. In both of the above examples, each recommendation of a resource allocation by the "fair arbitrator" effectively identifies a profile of "opportunities" or opportunity sets for the individuals involved. The crucial difference from standard axiomatic bargaining models in these examples is that the recommendation made by the arbitrator is not based on utilities of the individuals involved, but on opportunity sets that the recommended resource allocation may give rise to the involved individuals.

This departure from considerations of utilities of individuals to concerns of opportunity sets of individuals is well in line with the recent literature on opportunities and equality of opportunities. One branch of the literature is in political philosophy such as Sen (1980, 1985), Arneson (1989), and Cohen (1993), while the other is in economics, see, for example, Sen (2002), Pattanaik and Xu (1990), Kranich (1996), and Herrero (1997). In the latter branch of the literature, each individual is characterized by his opportunity sets, from which his well-being or welfare is evaluated.

An opportunity set of an individual is interpreted as a set of feasible options or alternatives available to the individual for living. Depending on the context, those alternatives can be commodity bundles, or bundles of characteristics à la Lancaster and Gorman, or bundles of functionings à la Sen (1980, 1985), and Nussbaum $(1988,1993,2000)$. A resource allocation in an economy then identifies a collection of opportunity sets, one for each individ- 
ual in the economy. Note that, for a given resource allocation, opportunity sets of individuals are necessarily interdependent. Note also that different resource allocations can give rise to various collections of opportunity sets for the individuals in the economy.

The question we address is, among various collections of opportunity sets for the individuals involved, how the "fair arbitrator" should make the recommendation on a resource allocation that yields a profile of opportunity sets for individuals in the economy, which is deemed as "fair." For this purpose, we extend standard bargaining models to the setting in which each individual is endowed with his opportunity sets, which are generated by his consumption bundles given his individual characteristics, and, in which the fair arbitrator makes recommendations based on profiles of opportunity sets for the individuals in the economy. ${ }^{1}$ We present two related formulations of extended bargaining models. In the first place, we formulate axioms in terms of profiles of opportunity sets. This formulation corresponds to standard bargaining models. The advantage of this formulation is to have a general and abstract framework to discuss bargaining problems in our setting. Since an important component of our primitive information about individuals is their opportunity sets, this formulation appeals directly to our intuition regarding this important component. To have a better understanding of the underlying allocations proposed by a solution to our extended bargaining models and with the above general formulation in hand, we next formulate our axioms in economic environments directly. For both formulations, we introduce the egalitarian solution for extended bargaining models and study it axiomatically.

The remainder of the paper is organized as follows. Section 2 introduces our economic environments and our problem. Section 3 defines and axiomatically characterizes the egalitarian solution in our context. Section 4 defines and axiomatically characterizes the egalitarian allocation rule in economic environments. We conclude the paper in Section 5 by briefly commenting on our approach and the results.

\footnotetext{
${ }^{1}$ Gotoh and Yoshihara (2003) discuss allocation mechanisms which assign individuals capability sets through distributing outputs produced by them. Their approach is quite different from the approach based on bargaining that this paper addresses.
} 


\section{Economic environments and bargaining prob- lems on opportunity assignments}

\subsection{Economic environments}

There are infinitely many types of goods (commodities). The universe of "potential goods" is denoted by $\Xi$, and the class of non-empty and finite subsets of $\Xi$ is designated by $\mathcal{M}$, with generic elements, $K, L, M, \ldots$, each is to be called a finite list of commodities. The cardinality of $M \in \mathcal{M}$ is denoted by $\# M=m$. For each $M \in \mathcal{M}$, let us denote a generic commodity bundle in $\mathbb{R}_{+}^{\# M}$ by $x$. To abuse the notation slightly, we shall write $\mathbb{R}_{+}^{\# M}$ as $\mathbb{R}_{+}^{m}$.

The population in the economy is given by the set $N=\{1, \cdots, n\}$, where $2 \leq n<+\infty$. Given a finite list of commodities $M \in \mathcal{M}$, every individual has a common consumption space $\mathbb{R}_{+}^{m}$. There are $k$ basic living conditions in the economy, which are relevant for all individuals for the purpose of describing their objective well-beings attainable by means of their consumption vectors. These basic living conditions can be interpreted broadly. For example, they can be skills that individuals can develop through education, or they can be occupations which individuals can engage in after the graduation at school. Or they can be characteristics of commodities in the sense of Gorman (1980) and Lancaster (1966), or they can be various functionings according to Sen $(1980,1985)$ and Nussbaum $(1988,1993,2000)$. For our formal analysis, we do not need to stick to a particular interpretation though a certain interpretation may be more appropriate than other interpretations for a given context.

Thus, an achievement of living condition $f$, where $f=1,2, \cdots, k$, by individual $i$ is denoted by $b_{i f} \in \mathbb{R}_{+}$. Individual $i$ 's achievement of basic living conditions is given by listing $b_{i f}: \mathbf{b}_{i}=\left(b_{i 1}, \cdots, b_{i k}\right) \in \mathbb{R}_{+}^{k}$. There are two crucial factors that determine the achievement of individual's basic living conditions: one is the amount of resources or commodities she can access for attaining these living conditions, and the other is the individual's ability to realize these living conditions by utilizing commodities. Note that, given $M \in \mathcal{M}$ and each individual $i \in N$, the latter is formulated as $i$ 's opportunity correspondence $c_{i}^{m}: \mathbb{R}_{+}^{m} \rightarrow \mathbb{R}_{+}^{k}$ which associates to every commodity vector

$x_{i} \in \mathbb{R}_{+}^{m}$ a non-empty subset $c_{i}^{m}\left(x_{i}\right)$ of $\mathbb{R}_{+}^{k}$. The intended interpretation is that $i$ is able to have access to each living-condition vector $\mathbf{b}_{i} \in c_{i}^{m}\left(x_{i}\right)$ by 
means of his commodity vector $x_{i}$. Each opportunity correspondence satisfies the following requirements:

(a) For all $x_{i}, x_{i}^{\prime} \in \mathbb{R}_{+}^{m}$ such that $x_{i} \leq x_{i}^{\prime}, c_{i}^{m}\left(x_{i}\right) \subseteq c_{i}^{m}\left(x_{i}^{\prime}\right)$ holds; $^{2}$

(b) For some $x_{i} \in \mathbb{R}_{+}^{m} \backslash\{\mathbf{0}\}, c_{i}^{m}\left(x_{i}\right) \cap \mathbb{R}_{++}^{k} \neq \varnothing$ holds, and $c_{i}^{m}(\mathbf{0})=\{\mathbf{0}\}$;

(c) For all $x_{i} \in \mathbb{R}_{+}^{m}, c_{i}^{m}\left(x_{i}\right)$ is compact and comprehensive in $\mathbb{R}_{+}^{k}$; and

(d) $c_{i}^{m}$ is continuous on $\mathbb{R}_{+}^{m}$ with respect to the Hausdorff topology.

Requirement (a) is a monotonicity property: more commodities generate "larger" opportunity sets. Requirement (b) essentially says that commodities are "desirable": they can help individuals in achieving positive levels of basic living conditions. Requirement (c ) stipulates that any given commodity bundle generates a bounded opportunity set. And finally, requirement (d) says that "small" changes in commodity bundles lead to "small" changes in opportunity sets.

Let $\mathfrak{C}^{M}$ be the set of all possible opportunity correspondences defined on $\mathbb{R}_{+}^{m}$, which satisfy the above (a), (b),(c) and (d). Given $M \in \mathcal{M}$, an economy with $\bar{x}$ endowments of $M$-goods is described by a list $\mathbf{e}=\left(M, \mathbf{c}^{m}, \bar{x}\right)=$ $\left(M,\left(c_{i}^{m}\right)_{i \in N}, \bar{x}\right)$, where $\mathbf{c}^{m} \in \mathfrak{C}^{M n}, \bar{x} \in \mathbb{R}_{+}^{m}$, and $\mathfrak{C}^{M n}$ stands for the $n$-fold Cartesian product of $\mathfrak{C}^{M}$. Let $\mathcal{E}^{M}$ be the class of all such economies with $\bar{x}$ endowments of $M$-goods. Let $\mathcal{E} \equiv \underset{M \in \mathcal{M}}{\cup} \mathcal{E}^{M}$. Given $\mathbf{e}=\left(M, \mathbf{c}^{m}, \bar{x}\right) \in \mathcal{E}^{M}$, a vector $\mathbf{x}=\left(x_{i}\right)_{i \in N} \in \mathbb{R}_{+}^{m n}$ is feasible for $\mathbf{e} \in \mathcal{E}^{M}$ if for all $i \in N, x_{i} \in \mathbb{R}_{+}^{m}$, and $\sum x_{i} \leq \bar{x}$. We denote by $A(\mathbf{e})$ the set of feasible allocations for $\mathbf{e} \in \mathcal{E}^{M}$. Let $A(\mathcal{E}) \equiv \cup_{\mathbf{e} \in \mathcal{E}} A(\mathbf{e})$.

For each individual $i \in N$, given $M \in \mathcal{M}$ and given $i$ 's consumption vector $x_{i}, c_{i}^{m}\left(x_{i}\right)$ generates a opportunity set $C_{i}=c_{i}^{m}\left(x_{i}\right)$ for $i$. An opportunity assignment is a list of $n$ opportunity sets one for each individual in the society. Given $\mathbf{e}=\left(M, \mathbf{c}^{m}, \bar{x}\right) \in \mathcal{E}$, the set of possible opportunity assignments for $\mathbf{e} \in \mathcal{E}$ is:

$\mathcal{C}(\mathbf{e}) \equiv\left\{\mathbf{C}=\left(C_{i}\right)_{i \in N} \subseteq \mathbb{R}_{+}^{k n} \mid \exists \mathbf{x}=\left(x_{i}\right)_{i \in N} \in A(\mathbf{e}): C_{i}=c_{i}^{m}\left(x_{i}\right)(\forall i \in N)\right\}$.

Note that for any $\mathbf{e}=\left(M, \mathbf{c}^{m}, \bar{x}\right) \in \mathcal{E}$, any $\mathbf{C}=\left(C_{i}\right)_{i \in N} \in \mathcal{C}(\mathbf{e})$, and any $i \in N$, the opportunity $C_{i}$ is a compact, comprehensive set in $\mathbb{R}_{+}^{k}$ containing the origin. For each $i \in N$ and each living condition $f=$ $1, \ldots, k$, let $\max _{f}\left(C_{i}\right)$ be the maximum amount of living condition $f$ by

\footnotetext{
${ }^{2}$ For all vectors $\mathbf{a}=\left(a_{1}, \ldots, a_{p}\right)$ and $\mathbf{b}=\left(b_{1}, \ldots, b_{p}\right) \in \mathbb{R}^{p}, \mathbf{a} \geq \mathbf{b}$ if and only if $a_{i} \geq b_{i}$ $(i=1, \ldots, p) ; \mathbf{a}>\mathbf{b}$ if and only if $\mathbf{a} \geq \mathbf{b}$ and $\mathbf{a} \neq \mathbf{b} ; \mathbf{a} \gg \mathbf{b}$ if and only if $a_{i}>b_{i}$ $(i=1, \ldots, p)$.
} 
$i$ that he can achieve under his opportunity set $C_{i}$; that is, $\max _{f}\left(C_{i}\right) \equiv$ $\max \left\{b_{f} \mid\left(b_{1}, \cdots, b_{f}, \cdots, b_{k}\right) \in C_{i}\right\}$. Let $\Sigma \equiv\{\mathcal{C} \mid \exists \mathbf{e} \in \mathcal{E}: \mathcal{C}=\mathcal{C}(\mathbf{e})\}$ be the class of all such possible sets of opportunity assignments. Note that each set $\mathcal{C}$ in $\Sigma$ is compact in terms of Hausdorff metric by the assumption (d) of the opportunity correspondence and the fact that $A(\mathbf{e})$ is compact for every $\mathbf{e} \in \mathcal{E}$. Also, for any $\mathcal{C} \in \Sigma$, if $\mathbf{C}=\left(C_{i}\right)_{i \in N} \in \mathcal{C}$, then for each $j \in N$, every living condition $f=1, \ldots, k$, and any $b_{f} \leq \max _{f}\left(C_{j}\right)$, there exists $\mathbf{C}^{\prime}=\left(C_{j}^{\prime}, \mathbf{C}_{-j}\right) \in \mathcal{C}$ such that $b_{f}=\max _{f}\left(C_{j}^{\prime}\right)$ and $C_{j}^{\prime} \subseteq C_{j}$ by the assumption of (a), (b), and (d) of opportunity correspondences.

\subsection{Opportunity sets and their ranking}

Let $\mathcal{K}$ be the universal class of compact, comprehensive subsets in $\mathbb{R}_{+}^{k}$ containing the origin. Thus, $C \in \mathcal{K}$ implies that for any $M \in \mathcal{M}$, there exists $c^{m} \in \mathfrak{C}^{M}$ such that for some $x \in \mathbb{R}_{+}^{m}, c^{m}(x)=C$. Note that for each $\mathcal{C} \in \Sigma$ and every $i \in N$, there exists $C_{i}^{*} \in \mathcal{K}$ such that for every $\mathbf{C} \in \mathcal{C}, C_{i}^{*} \supseteq C_{i}$ holds, and $\left(C_{i}^{*}, \mathbf{C}_{-i}^{0}\right) \in \mathcal{C}$ with $C_{j}^{0} \equiv\{\mathbf{0}\}$ for any $j \neq i$. This is followed from the requirement (a) of opportunity correspondences and the definition of $\Sigma$. Given $\mathcal{C} \in \Sigma$, let us denote such $C_{i}^{*}$ by $m_{i}(\mathcal{C})$ for each $i \in N$. Note that the profile $\left(m_{i}(\mathcal{C})\right)_{i \in N}$ is analogous to what is called in the standard bargaining theory the ideal point, which is necessary for defining the Kalai-Smorodinsky solution [Kalai and Smorodinsky (1975)].

How are various opportunity sets measured by individuals in the economy? We assume that there is an objective way of ranking various opportunity sets by individuals, where this objective measure of alternative opportunity sets is formalized as a binary relation $R \subseteq \mathcal{K} \times \mathcal{K}$. The relation $R$ satisfies reflexivity: [for all $C \in \mathcal{K},(C, C) \in R$ ], completeness: [for all $C, C^{\prime} \in \mathcal{K}$, $\left(C, C^{\prime}\right) \in R$ or $\left(C^{\prime}, C\right) \in R$ ], and transitivity: [for all $C, C^{\prime}, C^{\prime \prime} \in \mathcal{K}$, if $\left(C, C^{\prime}\right) \in R \&\left(C^{\prime}, C^{\prime \prime}\right) \in R$, then $\left.\left(C, C^{\prime \prime}\right) \in R\right]$. Thus, $R$ is an ordering over $\mathcal{K}$. Note $P$ and $I$ are respectively the asymmetric and symmetric parts of $R$.

For all $C, C^{\prime} \in \mathcal{K}$, we write $C>C^{\prime}$ if for all $\mathbf{b}^{\prime} \in C^{\prime}$, there exists $\mathbf{b} \in C$ such that $\mathbf{b} \gg \mathbf{b}^{\prime}$. Note that, given the comprehensiveness of opportunity sets in $\mathcal{K}$, when $C>C^{\prime}$, necessarily, we have $C^{\prime}$ as a proper subset of $C$.

In this paper, we assume that the ordering $R$ on $\mathcal{K}$ satisfies the following two properties:

Monotonicity: For all $C, C^{\prime} \in \mathcal{K}$, if $C \supseteq C^{\prime}$ then $\left(C, C^{\prime}\right) \in R$, and if $C>C^{\prime}$, then $\left(C, C^{\prime}\right) \in P$. 
Representability: There exists a real-valued, continuous function $G: \mathcal{K} \rightarrow$ $\mathbb{R}_{+}$such that for all $C, C^{\prime} \in \mathcal{K}$,

$$
G(C) \geq G\left(C^{\prime}\right) \Leftrightarrow\left(C, C^{\prime}\right) \in R .
$$

It may be noted that, in our context, Monotonicity is a fairly noncontroversial property and it essentially requires that a "bigger" opportunity set be ranked higher than a "smaller" opportunity set. Similar conditions have been used in the literature on ranking opportunity sets, see for example, Gaertner and Xu (2006), Pattanaik and Xu (2007), and Xu (2002, 2004). Representability requires the function $G$ representing the ordering $R$ to be continuous. See Pattanaik and Xu (2000), Xu (2004), and Savaglio and Vannucci (2006) for examples of such ordinal representations of a binary relation over opportunity sets.

\subsection{Bargaining problems on opportunity assignments}

The formal problem that we are interested in is the bargaining problem over opportunity assignments among individuals. Analogous to the standard bargaining model, we can interpret each $\mathcal{C} \in \Sigma$ as a bargaining problem and $\Sigma$ as the domain of bargaining problems, and a solution to the problem is to pick up a subset of opportunity assignments $\left\{\mathbf{C}=\left(C_{i}\right)_{i \in N}\right\}$ from $\mathcal{C}$. Then, a bargaining solution in this context is a correspondence $F$ which associates to every $\mathcal{C} \in \Sigma$, a non-empty subset $F(\mathcal{C}) \subseteq \mathcal{C}$.

How is our model related to the motivation discussed in the Introduction? The following examples may help us in understanding our approach.

Example 1: Let $k$ be the number of skills that an individual can develop through education, and let $x \in \mathbf{R}_{+}^{m}$ be an educational resource. Then, the $k$ dimensions of the opportunity set $\mathbf{c}_{i}^{m}(x) \subseteq \mathbb{R}_{+}^{k}$ represent the types of skills, and each element $\mathbf{b}_{i}=\left(b_{i f}\right)_{f \in\{1, \ldots, k\}} \in \mathbf{c}_{i}^{m}(x)$ implies that individual $i$ can develop the level of each skill $f$ up to $b_{i f}$, whenever he is educated with the educational support $x$ and some amount of his own effort. The difference of native talents among individuals is reflected in the difference of opportunity correspondences among them. In this setting, the bargaining problem would be to assign opportunities for future skills by allocating educational resources. 
Example 2: The WTO consists of many member countries and one of its functions is to settle disputes among its member countries. Disputes between or among member countries are really about net trades of goods, services or capital. The Dispute Settlement Body of the WTO thus makes recommendations as how to structure net trades among the affected member countries. ${ }^{3}$ Each member country is concerned about, for example, the aggregate employment rate, the growth rates of several sectors like manufacturing, agriculture, and service, and the health condition of its population. These concerns correspond to our notion of achievements. Each member country's interests can be captured by the country's opportunity sets representing opportunities to achieve a degree of employment rate, to have reasonable growth rates for its concerned sectors, and to offer its population a good health. The bargaining problem can then be interpreted as follows. The Dispute Settlement Body in the WTO mechanism acts as the fair arbitrator and it recommends the settlements that affect net trade based on equal opportunities for the disputed member countries along the factors that we discussed above.

Example 3: Our last example concerns the allocation of the budget by a central government to its several local jurisdictions. In many cases, the allocation of this budget intends for different localities to have equal opportunities for growth and for access to clean water, for example. Growth and access to clean water are two of the many factors that different local jurisdictions are concerned about, and local governments are concerned about their opportunities along these factors. The bargaining problem in this example can thus be viewed as how the fair arbitrator, the central government, makes budgetary allocations on the basis of equal opportunies for different local jurisdictions along those factors such as growth and environmental quality of each region.

\footnotetext{
${ }^{3}$ Quite often, disputes seemingly are about things like access to member countries' markets and information, legal protection concerning trades from member countries, or pricing rules. These are rules governing trade between and among nations and they have direct effect on net trade between member countries. As a consequence, we can interpret that disputes are really about net trade.
} 


\section{The egalitarian solution: a first character- ization}

Given a social evaluation of opportunity sets $R$ satisfying Monotonicity and Representability and its representation $G$, the egalitarian solution we consider in the paper is defined as follows:

Egalitarian Solution: $A$ bargaining solution $F^{E}$ is the egalitarian solution if and only if: for every $\mathcal{C} \in \Sigma, F^{E}(\mathcal{C})=\left\{\mathbf{C}=\left(C_{1}, \cdots, C_{n}\right) \in \mathcal{C}:\left(C_{i}, C_{j}\right) \in\right.$ $I$ holds for any $i, j \in N$ and there is no other $\mathbf{C}^{\prime} \in \mathcal{C}$ such that $\left(C_{i}^{\prime}, C_{i}\right) \in P$ for all $i \in N\}$.

Thus, the solution $F^{E}$ selects all the undominated assignments, such that in each of these assignments, everyone's opportunity is indifferent with any other's in terms of $R$.

\subsection{Axioms on bargaining solutions}

In this subsection, we shall present and discuss axioms on bargaining solutions over opportunity assignments. It may be remarked that most of the axioms introduced below are formulated in terms of opportunity assignments without explicitly referring to a given $G$ function representing the ordering $R$ over opportunity sets. Such axioms are considerably weak and can appeal to our intuitions directly.

The first axiom is the corresponding weak efficiency axiom in standard bargaining models.

Weak Efficiency (WE): For each $\mathcal{C} \in \Sigma$ and each $\mathbf{C}=\left(C_{i}\right)_{i \in N} \in F(\mathcal{C})$, there is no $\mathbf{C}^{\prime}=\left(C_{i}^{\prime}\right)_{i \in N} \in \mathcal{C}$ such that for every $i \in N, C_{i}^{\prime}>C_{i}$.

Therefore, the axiom (WE) requires that the solution should not select an opportunity assignment that is strictly dominated by another feasible opportunity assignment. It may be reminded that, when an opportunity assignment $\mathcal{C}^{\prime}$ strictly dominates an opportunity assignment $\mathcal{C}$, we have $\left[C_{i}^{\prime}>C_{i}\right.$ for every $i \in N]$, which requires that, for each $i \in N, C_{i}^{\prime}$ is obtained from $C_{i}$ by expanding it "outwardly"; as a consequence, necessarily, each $C_{i}$ is a proper subset of $C_{i}^{\prime}$. 
To introduce our next axiom, we first define a symmetric problem. We say that $\mathcal{C} \in \Sigma$ is symmetric if for every permutation $\pi: N \rightarrow N$, and for every $\mathbf{C}=\left(C_{i}\right)_{i \in N} \in \mathcal{C}, \pi(\mathbf{C}) \equiv\left(C_{\pi(i)}\right)_{i \in N} \in \mathcal{C}$ holds.

Symmetry (S): For each $\mathcal{C} \in \Sigma$, if (i) $\mathcal{C}$ is symmetric, and (ii) there exists $a \mathbf{C} \in \mathcal{C}$ such that it is weakly efficient in $\mathcal{C}$ and $C_{i}=C_{j}$ for all $i, j \in N$, then there exists some $\mathbf{C}^{*} \in F(\mathcal{C})$ such that $C_{i}^{*}=C_{j}^{*}$ for all $i, j \in N$, and there is no $\mathbf{C}^{\prime} \in F(\mathcal{C})$ such that $C_{i}^{\prime}>C_{j}^{\prime}$ for some $i, j \in N$.

The axiom (S) stipulates that, for each symmetric problem containing problems with identical opportunity assignments, the solution selects at least one identical opportunity assignment if there is an identical opportunity assignment which is also weakly efficient in the problem, and further, no opportunity assignment selected by the solution is such that one individual's opportunity set strictly dominates another individual's opportunity set.

The following axiom is analogous to the axiom of contraction independence in standard bargaining models:

Contraction Independence (CI): For each $\mathcal{C}, \mathcal{C}^{\prime} \in \Sigma$ with $\mathcal{C} \supseteq \mathcal{C}^{\prime}$, if $F(\mathcal{C}) \cap \mathcal{C}^{\prime} \neq \varnothing$, then $F\left(\mathcal{C}^{\prime}\right)=F(\mathcal{C}) \cap \mathcal{C}^{\prime}$.

The axiom (CI) corresponds to Nash's Independence of Irrelevant Alternatives in standard bargaining models. It requires that if an opportunity assignment is chosen from a "larger" problem and is still available when the larger problem shrinks to a smaller problem, then it should be chosen from the smaller problem as well.

Our final axiom is an informational requirement on a solution to a problem and is stated below:

Informational Invariance (II) : For each $\mathcal{C} \in \Sigma$ and each $\mathbf{C}=\left(C_{i}\right)_{i \in N}, \mathbf{C}^{\prime}=$ $\left(C_{i}^{\prime}\right)_{i \in N} \in \mathcal{C}$, if $\mathbf{C} \in F(\mathcal{C})$ and $\left(C_{i}^{\prime}, C_{i}\right) \in I$ for all $i \in N$, then $\mathbf{C}^{\prime} \in F(\mathcal{C})$.

According to the axiom (II), if two opportunity assignments are "equivalent" in the sense that the two opportunity sets for each and every individual specified by the corresponding opportunity assignments are ranked equally, then whenever one opportunity assignment is chosen by the solution, the other opportunity assignment should be chosen by the solution as well. The axiom (II) thus implies that the informational requirement in our context is contained exclusively in the social evaluation ordering $R$. A similar axiom, 
called No Discrimination, ${ }^{4}$ is discussed by Thomson (1983) in the context of fair allocation problems.

\subsection{A characterization of the egalitarian solution}

Before we present our characterization result, the following observations are useful throughout this subsection. Let $G$ be the real-valued, ordinal representation of the social ordering $R$. For each bargaining problem $\mathcal{C} \in \Sigma$, we define

$$
G(\mathcal{C}) \equiv\left\{G(\mathbf{C})=\left(G\left(C_{i}\right)\right)_{i \in N} \in \mathbb{R}_{+}^{n} \mid \mathbf{C} \in \mathcal{C}\right\} .
$$

Let $\partial G(\mathcal{C})$ be the upper boundary of $G(\mathcal{C})$. Since $\mathcal{C}$ is derived from an underlying economic environment $\mathbf{e}=\left(M, \mathbf{c}^{m}, \bar{x}\right) \in \mathcal{E}$, where $\mathbf{c}^{m}$ is a profile of opportunity correspondences satisfying the requirements (a), (b), (c), and (d), and $G$ is continuous on $\mathcal{K}, \partial G(\mathcal{C})$ constitutes a connected set in $\mathbb{R}_{+}^{n}$. Moreover, since $\mathcal{C}$ is comprehensive ${ }^{5}$ by the requirements (a), (b), and (d) of opportunity correspondences, $G(\mathcal{C})$ must be comprehensive. Finally, by choosing $G(\{\mathbf{0}\})=0$ for the zero vector $\mathbf{0} \in \mathbb{R}_{+}^{k}, G(\mathcal{C})$ has $\mathbf{0} \in \mathbb{R}_{+}^{n}$ as its element, since $\underbrace{(\{\mathbf{0}\}, \ldots,\{\mathbf{0}\})}_{n \text {-times }} \in \mathcal{C}$. Therefore, $G(\mathcal{C})$ corresponds to a standard normalized, non-convex, and comprehensive bargaining problem.

Given these observations, we can easily see that the solution $F^{E}$ is welldefined in the sense that for each $\mathcal{C} \in \Sigma, F^{E}(\mathcal{C})$ is non-empty. This is because, for each $\mathcal{C} \in \Sigma$, its corresponding $\partial G(\mathcal{C})$ always contains the vector of equal real numbers, and the inverse image of this vector constitutes the set $F^{E}(\mathcal{C})$. Thus, our next concern is to discuss a characterization of this solution.

Theorem 1: The egalitarian solution $F^{E}$ is the unique solution satisfying (WE), (S), (CI) and (II).

Proof. First, it may be checked that $F^{E}$ satisfies the four axioms of the theorem.

\footnotetext{
${ }^{4}$ No Discrimination requires that if, for any allocation recommended by a solution, there exists another allocation whose corresponding utility allocation is identical to that of the first allocation, then the second allocation should be recommended by the solution as well.

${ }^{5} \mathcal{C}$ is comprehensive if, for each $\mathbf{C} \in \mathcal{C}$ and each $i \in N$ with $C_{i} \neq\{\mathbf{0}\}$, there exists $\mathbf{C}^{\prime}$ $\in \mathcal{C}$ such that $C_{i}^{\prime}<C_{i}$ and $C_{j}^{\prime} \subseteq C_{j}$ for all $j \in N \backslash\{i\}$.
} 
Next, we show that if a solution $F$ satisfies (WE), (S), (CI) and (II), then $F=F^{E}$. Consider a bargaining problem $\mathcal{C} \in \Sigma$, which is derived from an underlying economic environment $\mathbf{e}=\left(M, \mathbf{c}^{m}, \bar{x}\right) \in \mathcal{E}$. Suppose $F \neq F^{E}$. By $(\mathrm{II}), G(F(\mathcal{C})) \neq G\left(F^{E}(\mathcal{C})\right)$. Then, there exists $\mathbf{r}^{*} \in G(F(\mathcal{C})) \backslash G\left(F^{E}(\mathcal{C})\right)$. For each $i \in N$, let

$$
\mathcal{C}(i) \equiv\left\{C_{i}^{\prime} \in \mathcal{K} \mid \exists \mathbf{C}_{-i} \in \mathcal{K}^{n-1}:\left(C_{i}^{\prime}, \mathbf{C}_{-i}\right) \in \mathcal{C}\right\},
$$

and for each $r \in \mathbb{R}_{+}$, define

$$
\mathcal{C}(i ; r) \equiv\left\{C_{i}^{\prime} \in \mathcal{C}(i) \mid G\left(C_{i}^{\prime}\right)=r\right\} .
$$

Consider $\operatorname{comp}\left\{\mathbf{r}^{*}\right\} \equiv\left\{\mathbf{r} \in \mathbb{R}_{+}^{n}: \mathbf{r} \leq \mathbf{r}^{*}\right\}$, and $\mathcal{C}^{*} \equiv G^{-1}\left(\operatorname{comp}\left\{\mathbf{r}^{*}\right\}\right)$.

Insert Figure 1 around here.

Since $G$ is continuous, we can choose a subset $\mathcal{C}_{s}^{*} \subseteq \mathcal{C}^{*}$ so that (i) for each $\mathbf{r} \in \operatorname{comp}\left\{\mathbf{r}^{*}\right\}$, there exists a unique $\left(C_{i}^{r_{i}}\right)_{i \in N} \in \mathcal{C}_{s}^{*}$ such that $\left[C_{i}^{r_{i}} \in \mathcal{C}\left(i ; r_{i}\right)\right.$ for all $i \in N]$; (ii) for any $\mathbf{r}, \mathbf{r}^{\prime} \in \operatorname{comp}\left\{\mathbf{r}^{*}\right\}$, and each $i \in N, C_{i}^{r_{i}}=C_{i}^{r_{i}^{\prime}}$ holds if $r_{i}=r_{i}^{\prime}$, and $C_{i}^{r_{i}}>C_{i}^{r_{i}^{\prime}}$ holds if $r_{i}>r_{i}^{\prime}$; and (iii) for any $\mathbf{r}^{\prime} \in \operatorname{comp}\left\{\mathbf{r}^{*}\right\}$, $\lim _{\mathbf{r} \rightarrow \mathbf{r}^{\prime}}\left(C_{i}^{r_{i}}\right)_{i \in N}=\left(C_{i}^{r_{i}^{\prime}}\right)_{i \in N}$ holds. By condition (i) of the definition of $\mathcal{C}_{s}^{*}$, $G\left(\mathcal{C}_{s}^{*}\right)=\operatorname{comp}\left\{\mathbf{r}^{*}\right\}$. Note that (i) requires that for each $\mathbf{r} \in \operatorname{comp}\left\{\mathbf{r}^{*}\right\}$, there is a unique assignment in $\mathcal{C}_{s}^{*}$ such that its corresponding $G$-values are $\mathbf{r}$; (ii) requires that for any $r_{i}, r_{i}^{\prime}$ of $\mathbf{r}, \mathbf{r}^{\prime} \in \operatorname{comp}\left\{\mathbf{r}^{*}\right\}$, their corresponding opportunity sets are either identical or related to each other by set-inclusion domination; and (iii) requires that $\mathcal{C}_{s}^{*}$ is a closed set.

Now, by using the information of $\mathcal{C}_{s}^{*}$, let us construct a new economy $\mathbf{e}^{*}=\left(M^{*}, \widehat{\mathbf{c}}^{1}, \bar{x}^{*}\right) \in \mathcal{E}$. Firstly, let $M^{*} \cap M=\varnothing, \# M^{*}=1$, and $\bar{x}^{*}=1$. Secondly, for each $i \in N$, let the opportunity correspondence $\widehat{c}_{i}^{1}$ be given as follows:

(i) for all $x \in\left[0, \frac{1}{n}\right], \widehat{c}_{i}^{1}(x)=C_{i}^{r_{i}^{\prime}}$ with $r_{i}^{\prime}=n x \cdot r_{i}^{*}$; and

(ii) for all $x \in\left(\frac{1}{n}, 1\right], \widehat{c}_{i}^{1}(x)=C_{i}^{r_{i}^{*}}$.

Then, consider $\mathcal{C}^{* *} \equiv \mathcal{C}\left(\mathbf{e}^{*}\right) \in \Sigma$. Since $\mathcal{C}^{* *} \subseteq \mathcal{C}^{*} \subseteq \mathcal{C}$ and $G\left(\mathcal{C}^{* *}\right)=G\left(\mathcal{C}^{*}\right)$, we obtain $\mathbf{r}^{*} \in G\left(F\left(\mathcal{C}^{* *}\right)\right)$ from $(\mathrm{CI})$. Next, consider $\mathcal{C}^{\triangle} \equiv \cup_{\pi \in \Pi} \pi\left(\mathcal{C}^{* *}\right)$.

Insert Figure 2 around here.

In the following discussion, we will construct a new economy $\mathbf{e}^{\triangle} \in \mathcal{E}$ such that $\mathcal{C}\left(\mathbf{e}^{\triangle}\right) \supseteq \mathcal{C}^{\triangle}$ and $G\left(\mathcal{C}\left(\mathbf{e}^{\triangle}\right)\right)=G\left(\mathcal{C}^{\triangle}\right)$. Firstly, given $\mathbf{r}^{*} \in G\left(\mathcal{C}^{\triangle}\right)$, 
let $r_{\max }^{*} \equiv \max _{i \in N}\left(r_{i}^{*}\right)$ and $r_{\min }^{*} \equiv \min _{i \in N}\left(r_{i}^{*}\right)$. Define, for each $i \in N$, an opportunity correspondence $\widehat{c}_{i}^{* 1}:[0,1] \rightarrow \mathbb{R}_{+}^{k}$ by:

(I) for all $x \in\left[0, \frac{1}{n}\right], \widehat{c}_{i}^{* 1}(x)=C_{i}^{r_{i}^{\prime}}$ with $r_{i}^{\prime}=n x \cdot r_{\min }^{*}$; and

(II) for all $x \in\left(\frac{1}{n}, 1\right], \widehat{c}_{i}^{* 1}(x)=C_{i}^{r_{i}^{\prime}}$ with $r_{i}^{\prime}=\frac{n\left(r_{i}^{*}-r_{\min }^{*}\right)}{n-1} x+\frac{n r_{\min }^{*}-r_{i}^{*}}{n-1}$, where $C_{i}^{r_{i}^{\prime}}$, for each $i \in N$, comes from $\mathcal{C}^{* *}$.

Secondly, let us define $c^{\triangle n}:[0,1]^{n} \rightarrow \mathbb{R}_{+}^{k}$ by $c^{\triangle n}(x) \equiv \cup_{i \in N} \widehat{c}_{i}^{* 1}\left(x_{i}\right)$ for each $x=\left(x_{i}\right)_{i \in N} \in[0,1]^{n}$. Thirdly, define $\mathbf{e}^{\triangle} \equiv\left(M^{*(n)}, \mathbf{c}^{\triangle n},\left(\bar{x}^{*}\right)^{n}\right) \in \mathcal{E}$, where $M^{*(n)} \cap M=\varnothing$ and \# $M^{*(n)}=n, \mathbf{c}^{\triangle n}=\underbrace{\left(c^{\Delta n}, \ldots, c^{\triangle n}\right)}_{n \text {-times }}$, and $\left(\bar{x}^{*}\right)^{n} \equiv \underbrace{(1, \ldots, 1)}_{n \text {-times }}$.

Note that for each $x=\left(x_{i}\right)_{i \in N} \in[0,1]^{n}$, if $x=\left(x_{i}, \mathbf{0}_{-i}\right), c^{\triangle n}(x)=\widehat{c}_{i}^{* 1}\left(x_{i}\right)$, and in particular if $x_{i}=\frac{1}{n}$, then $c^{\triangle n}(x)=C_{i}^{r_{\text {min }}^{*}}$, whereas if $x_{i}=1$, then $c^{\triangle n}(x)=C_{i}^{r_{i}^{*}} ;$ if $x=\underbrace{\left(\frac{1}{n}, \ldots, \frac{1}{n}\right)}_{n \text {-times }}$, then $c^{\triangle n}(x)=\cup_{i \in N} C_{i}^{r_{\text {min }}^{*}} \equiv C^{r_{\text {min }}^{*}}$; if $x$ is such that $x_{i}=1$ for some $i \in N$, then $G\left(c^{\Delta n}(x)\right) \geq r_{i}^{*}$; and if $x=\left(\bar{x}^{*}\right)^{n}$, then $c^{\triangle n}(x)=\cup_{i \in N} C_{i}^{r_{i}^{*}}$ and $G\left(c^{\triangle n}(x)\right)=r_{\max }^{*}$. By these properties followed from the definition of $c^{\triangle n}$, we can see that $\mathcal{C}\left(\mathbf{e}^{\triangle}\right) \supseteq \mathcal{C}^{\triangle}$ and $G\left(\mathcal{C}\left(\mathbf{e}^{\triangle}\right)\right)=G\left(\mathcal{C}^{\triangle}\right)$. Moreover, $\mathcal{C}\left(\mathbf{e}^{\triangle}\right)$ is a symmetric problem containing a weakly efficient identical opportunity assignment $\mathbf{C}^{\mathbf{r}_{\min }^{*}} \equiv \underbrace{\left(C^{r_{\min }^{*}}, \ldots, C^{r_{\min }^{*}}\right)}_{n \text {-times }}$.

From the construction of $\mathcal{C}\left(\mathbf{e}^{\triangle}\right)$, by (WE), (S), and (II), we must have $G\left(F\left(\mathcal{C}\left(\mathbf{e}^{\triangle}\right)\right)\right)=\left\{G\left(\mathbf{C}^{\mathbf{r}_{\text {min }}^{*}}\right)\right\}$. Let $\mathbf{r}^{\triangle E} \equiv G\left(\mathbf{C}^{\mathbf{r}_{\text {min }}^{*}}\right)$, which is the egalitarian outcome for the problem $\mathcal{C}\left(\mathbf{e}^{\triangle}\right)$.

Insert Figure 3 around here.

Since $\mathbf{r}^{\triangle E} \in \partial G\left(\mathcal{C}^{* *}\right)$, there exists $\left(C_{i}^{r_{\text {min }}^{*}}\right)_{i \in N} \in \mathcal{C}^{* *}$ such that $G\left(\left(C_{i}^{r_{\text {min }}^{*}}\right)_{i \in N}\right)=$ $\mathbf{r}^{\triangle E}$. Thus, by $(\mathrm{CI}),\left\{\mathbf{r}^{\triangle E}\right\}=G\left(F\left(\mathcal{C}^{* *}\right)\right)$, which is a desired contradiction, since $\mathbf{r}^{*} \in G\left(F\left(\mathcal{C}^{* *}\right)\right)$.

Insert Figure 4 around here.

Thus, $G(F(\mathcal{C})) \backslash G\left(F^{E}(\mathcal{C})\right)=\varnothing$. By (II), clearly, $F=F^{E}$.

It is easy to check the independence of axioms in Theorem 1, so we only note the results and omit the detailed proofs here: Regarding (II), any proper 
subsolution of $F^{E}$ satisfies all the axioms except (II); regarding (WE), a solution which solely selects $\underbrace{(\{\mathbf{0}\}, \ldots,\{\mathbf{0}\})}_{n \text {-times }}$ from any bargaining problem satisfies all the axioms but (WE); regarding (S), the Nash solution which is introduced by $\mathrm{Xu}$ and Yoshihara (2006a) for (opportunity)-bargaining problems satisfies all the axioms except (S); and regarding (CI), the Kalai-Smorodinsky solution which is introduced by Xu and Yoshihara (2006a) for (opportunity)bargaining problems satisfies all the axioms but (CI).

Note that the (utility)-egalitarian solution to standard convex and comprehensive (utility)-bargaining problems was axiomatically studied by Kalai (1977), where the solution is characterized by weak efficiency, symmetry, and strong monotonicity. Our result of Theorem 1 suggests that the (utility)egalitarian solution to standard non-convex and comprehensive utility-bargaining problems can be characterized by the corresponding axioms of weak efficiency, symmetry and contraction independence. This indeed is the case, see $\mathrm{Xu}$ and Yoshihara (2006). It may be noted that, in the standard convex (resp. nonconvex) and comprehensive (utility)-bargaining problems, the corresponding axiom to our axiom (II) becomes redundant.

\section{The egalitarian allocation rule in economic environments}

Though Theorem 1 gives us a good general understanding of the egalitarian solution to bargaining problems in our setting, it does not say anything directly about properties of resource allocation mechanisms which realize the egalitarian opportunity assignments in each economy. Quite often, it would be useful to know properties of such mechanisms when the egalitarian solution is applied to the concrete bargaining problems on economic resource allocations. Moreover, in the context of fair allocation problems, characterizing such mechanisms would be useful as well if the egalitarian opportunity assignments are deemed to be desirable outcomes. For these purposes, in this section, we reformulate our bargaining problems directly in economic environments. ${ }^{6}$ We start the analysis by introducing some additional definitions.

An allocation rule is a correspondence $\varphi$ which associates to every $\mathbf{e} \in \mathcal{E}$,

\footnotetext{
${ }^{6} \mathrm{~A}$ parallel analysis was also developed in the standard (utility)-bargaining problems by Roemer (1988) and Yoshihara (2003, 2006).
} 
a non-empty subset $\varphi(\mathbf{e}) \subseteq A(\mathbf{e})$. An allocation rule $\varphi$ attains a bargaining solution $F$ if and only if for every e $\in \mathcal{E}, \mathbf{c}(\varphi(\mathbf{e}))=F(\mathcal{C}(\mathbf{e}))$, where $\mathbf{c}(\varphi(\mathbf{e})) \equiv$ $\left\{\mathbf{C}=\left(C_{i}\right)_{i \in N} \in \mathcal{C}(\mathbf{e}) \mid \exists \mathbf{x}=\left(x_{i}\right)_{i \in N} \in \varphi(\mathbf{e}): C_{i}=c_{i}^{m}\left(x_{i}\right)(\forall i \in N)\right\}$. Then, the egalitarian allocation rule is introduced as follows.

Egalitarian Allocation Rule: An allocation rule $\varphi^{E}$ is the egalitarian rule if it attains the egalitarian solution: for all $\mathbf{e} \in \mathcal{E}, \mathbf{c}\left(\varphi^{E}(\mathbf{e})\right)=F^{E}(\mathcal{C}(\mathbf{e}))$.

We now present and discuss relevant axioms on allocation rules that attain bargaining solutions over opportunity assignments. We first introduce an axiom that is similar to weak efficiency in standard bargaining models. Its intuition is straightforward.

Weak Economic Efficiency (WEE): For each $\mathbf{e}=\left(M, \mathbf{c}^{m}, \bar{x}\right) \in \mathcal{E}$ and each $\mathbf{x} \in \varphi(\mathbf{e})$, there is no $\mathbf{x}^{\prime} \in A(\mathbf{e})$ such that for every $i \in N, c_{i}^{m}\left(x_{i}^{\prime}\right)>$ $c_{i}^{m}\left(x_{i}\right)$.

We shall denote the set of weakly economic efficient allocations for e by $W E(\mathbf{e})$.

The next two axioms correspond to the axioms of symmetry and contraction independence introduced in Section 3.

Economic Symmetry (ES): For each $\mathbf{e}=\left(M, \mathbf{c}^{m}, \bar{x}\right) \in \mathcal{E}$ with $c_{i}^{m}=c_{j}^{m}$ for all $i, j \in N$, if $\mathbf{x} \in \varphi(\mathbf{e})$, then for any $i, j \in N, c_{i}^{m}\left(x_{i}\right)=c_{j}^{m}\left(x_{j}\right)$.

Economic Contraction Independence (ECI): For each $\mathbf{e}=\left(M, \mathbf{c}^{m}, \bar{x}\right), \mathbf{e}^{\prime}=$ $\left(M, \mathbf{c}^{m}, \bar{x}^{\prime}\right) \in \mathcal{E}$ with $\bar{x} \geq \bar{x}^{\prime}$, if $\mathbf{c}(\varphi(\mathbf{e})) \cap \mathcal{C}\left(\mathbf{e}^{\prime}\right) \neq \varnothing$, then $\mathbf{c}\left(\varphi\left(\mathbf{e}^{\prime}\right)\right)=$ $\mathbf{c}(\varphi(\mathbf{e})) \cap \mathcal{C}\left(\mathbf{e}^{\prime}\right)$.

Informational requirements on allocation rules in the current setting are stated in the following axioms.

Strong Economic Informational Invariance (SEII) : For each $\mathbf{e}=$ $\left(M, \mathbf{c}^{m}, \bar{x}\right), \mathbf{e}^{\prime}=\left(L, \mathbf{c}^{l}, \bar{x}^{\prime}\right) \in \mathcal{E}$ with $\mathcal{C}(\mathbf{e})=\mathcal{C}\left(\mathbf{e}^{\prime}\right)$, for any $\mathbf{x} \in \varphi(\mathbf{e})$ and any $\mathbf{x}^{\prime} \in A\left(\mathbf{e}^{\prime}\right)$, if $\left(c_{i}^{l}\left(x_{i}^{\prime}\right), c_{i}^{m}\left(x_{i}\right)\right) \in I$ holds for all $i \in N$, then $\mathbf{x}^{\prime} \in \varphi\left(\mathbf{e}^{\prime}\right)$.

Economic Informational Invariance (EII) : For each $\mathbf{e}=\left(M, \mathbf{c}^{m}, \bar{x}\right) \in$ $\mathcal{E}$, for any $\mathbf{x} \in \varphi(\mathbf{e})$ and any $\mathbf{x}^{\prime} \in A(\mathbf{e})$, if $\left(c_{i}^{m}\left(x_{i}^{\prime}\right), c_{i}^{m}\left(x_{i}\right)\right) \in I$ holds for all $i \in N$, then $\mathbf{x}^{\prime} \in \varphi(\mathbf{e})$. 
Full Correspondence $(\mathbf{F}):$ For each $\mathbf{e}=\left(M, \mathbf{c}^{m}, \bar{x}\right) \in \mathcal{E}$, for any $\mathbf{x} \in \varphi(\mathbf{e})$ and any $\mathbf{x}^{\prime} \in A(\mathbf{e})$, if $c_{i}^{m}\left(x_{i}^{\prime}\right)=c_{i}^{m}\left(x_{i}\right)$ holds for all $i \in N$, then $\mathbf{x}^{\prime} \in \varphi(\mathbf{e})$.

It may be noted that (SEII) implies (EII) and (EII) implies (F). (SEII) requires that, for any two economies having the same set of opportunity assignments, if an allocation $\mathbf{x}$ is chosen by the allocation rule for the first economy and if an allocation $\mathbf{x}^{\prime}$ is feasible in the second economy, then the allocation $\mathbf{x}^{\prime}$ should be chosen for the second economy as long as every individual views the opportunity set generated under $\mathbf{x}$ being indifferent to the opportunity set generated under $\mathbf{x}^{\prime}$. (EII) is weaker than (SEII) in that (EII) is confined to the same economy, and (F) is weaker than (EII) in that the allocation $\mathbf{x}^{\prime}$ should be chosen for the second economy as long as (i) $\mathbf{x}$ is chosen for the first economy, (ii) $\mathbf{x}^{\prime}$ is feasible, and (iii) $\mathbf{x}^{\prime}$ generates the same opportunity set for every individual as $\mathbf{x}$.

(SEII) can be further decomposed. It turns out that (SEII) embodies an element relating to dimensional changes in endowments of commodities. To capture this idea formally, we introduce a definition first. Letting $x \in \mathbb{R}_{+}^{m}$ and $c_{i}^{m} \in \mathfrak{C}^{M}$ and letting $K$ be a proper subset of $M$, we say that each good in $K$ is useless for individual $i \in N$ at $x$ if, for all $x_{K}^{\prime} \equiv\left(x_{f}^{\prime}\right)_{f \in K} \in$ $\mathbb{R}_{+}^{k}, c_{i}^{m}\left(x_{K}^{\prime}, x_{M \backslash K}\right)=c_{i}^{m}\left(x_{K}, x_{M \backslash K}\right)$, where $x_{K} \equiv\left(x_{f}\right)_{f \in K}$. Therefore, a commodity is useless for an individual at a commodity bundle $x$ if it does not "contribute" anything to this individual's opportunity set under $x$.

Independence of Useless New Commodities (INC): Let $\mathbf{e}=\left(M, \mathbf{c}^{m}, \bar{x}\right) \in$ $\mathcal{E}^{M}$, and let $\mathbf{e}^{\prime}=\left(M \cup L, \mathbf{c}^{m+l},(\bar{x}, \bar{y})\right) \in \mathcal{E}^{M \cup L}$, where $M \cap L=\varnothing$, be such that (1) for any $\mathbf{x}=\left(x_{i}\right)_{i \in N} \in W E(\mathbf{e})$, there exists $\left(y_{i}^{\mathbf{x}}\right)_{i \in N} \in \mathbb{R}_{+}^{n l}$ such that

$$
c_{i}^{m+l}\left(x_{i}, y_{i}^{\mathbf{x}}\right)=c_{i}^{m}\left(x_{i}\right)(\forall i \in N) \text { and }\left(x_{i}, y_{i}^{\mathbf{x}}\right)_{i \in N} \in W E\left(\mathbf{e}^{\prime}\right),
$$

and (2) there exists $\widehat{\mathbf{x}}=\left(\widehat{x}_{i}\right)_{i \in N} \in W E(\mathbf{e})$ such that each good of $L$ is useless for every agent $i \in N$ at $\left(\widehat{x}_{i}, \mathbf{0}\right) \in \mathbb{R}_{+}^{m+l}$. Then, $\widehat{\mathbf{x}} \in \varphi(\mathbf{e})$ if and only if $\left(\widehat{x}_{i}, \mathbf{0}\right)_{i \in N} \in \varphi\left(\mathbf{e}^{\prime}\right)$.

(INC) essentially requires that, by adding useless new commodities to an economy, those allocations that "preserve" the original allocations chosen by an allocation rule for the original economy and use none of the useless new commodities should continue to be chosen from the "enlarged" economy by the allocation rule. 
The characterization of the egalitarian allocation rule is summarized in the following theorem, Theorem 2.

Theorem 2: The egalitarian allocation rule $\varphi^{E}$ is the unique rule satisfying (WEE), (ES), (ECI), (EII), and (INC).

To prove Theorem 2, we first prove the following series of lemmas. The proof of Theorem 2 will then follow. In Lemma 1, we shall establish the following result: Given any two economies that generate the same set of possible opportunity assignments, if an allocation rule satisfies (WEE), (F) and (INC), then the sets of all opportunity assignments attained by the allocations chosen by the allocation rule under the two economies must be the same. Formally:

Lemma 1: Let $\mathbf{e}_{1}, \mathbf{e}_{2} \in \mathcal{E}$ be such that $\mathbf{e}_{1}=\left(M, \mathbf{c}^{m}, \bar{x}\right) \in \mathcal{E}^{M}, \mathbf{e}_{2}=$ $\left(L, \mathbf{c}^{l}, \bar{y}\right) \in \mathcal{E}^{L}$, and $\mathcal{C}\left(\mathbf{e}_{1}\right)=\mathcal{C}\left(\mathbf{e}_{2}\right)$. Then, the allocation rule $\varphi$ which satisfies (WEE), (F), and (INC) has the following property: $\mathbf{c}\left(\varphi\left(\mathbf{e}_{1}\right)\right)=\mathbf{c}\left(\varphi\left(\mathbf{e}_{2}\right)\right)$.

Proof. Let $\mathbf{e}_{1}, \mathbf{e}_{2} \in \mathcal{E}$ be such that $\mathbf{e}_{1}=\left(M, \mathbf{c}^{m}, \bar{x}\right) \in \mathcal{E}^{M}, \mathbf{e}_{2}=\left(L, \mathbf{c}^{l}, \bar{y}\right) \in$ $\mathcal{E}^{L}$, and $\mathcal{C}\left(\mathbf{e}_{1}\right)=\mathcal{C}\left(\mathbf{e}_{2}\right)=\mathcal{C}$. In the following, we will construct another economy $\mathbf{e}_{3}=\left(S, \mathbf{c}^{s}, \bar{z}\right) \in \mathcal{E}^{S}$ such that $s=m+l$ and $\mathcal{C}\left(\mathbf{e}_{3}\right)=\mathcal{C}\left(\mathbf{e}_{1}\right)=$ $\mathcal{C}\left(\mathbf{e}_{2}\right)$. Then, it will be shown that (WEE), (F), and (INC) imply $\mathbf{c}\left(\varphi\left(\mathbf{e}_{1}\right)\right)=$ $\mathbf{c}\left(\varphi\left(\mathbf{e}_{3}\right)\right)$. After showing this claim, we will show that (WEE), (F), and (INC) imply $\mathbf{c}\left(\varphi\left(\mathbf{e}_{2}\right)\right)=\mathbf{c}\left(\varphi\left(\mathbf{e}_{3}\right)\right)$. Then, $\mathbf{c}\left(\varphi\left(\mathbf{e}_{1}\right)\right)=\mathbf{c}\left(\varphi\left(\mathbf{e}_{2}\right)\right)$ will follow easily.

Let $S$ be a set of commodities such that $\# S=s \equiv m+l$. For each $\mathbf{C} \in \mathcal{C}$, there exist $\mathbf{x} \in A\left(\mathbf{e}_{1}\right)$ and $\mathbf{y} \in A\left(\mathbf{e}_{2}\right)$ such that $c_{i}^{m}\left(x_{i}\right)=C_{i}=c_{i}^{l}\left(y_{i}\right)$ for each $i \in N$. This property implies that, for each $i \in N$, there exists a bijection $\tau_{i}:[0, \bar{x}] \rightarrow[0, \bar{y}]$ such that for each $x \in[0, \bar{x}], c_{i}^{m}(x)=c_{i}^{l}\left(\tau_{i}(x)\right)$, and for each $y \in[0, \bar{y}], c_{i}^{l}(y)=c_{i}^{m}\left(\tau_{i}^{-1}(y)\right)$. We then define, for each $i \in N$ and each $(x, y) \in \mathbb{R}_{+}^{s}$,

$$
\begin{aligned}
c_{i}^{*}(x, y) & =c_{i}^{m}\left(x \wedge \tau_{i}^{-1}(y)\right) \text { if }(x, y) \leq(\bar{x}, \bar{y}) \\
c_{i}^{*}(x, y) & =c_{i}^{m}(x) \text { otherwise, } \\
\text { where } x \wedge x^{\prime} & \equiv\left(\min \left\{x_{h}, x_{h}^{\prime}\right\}\right)_{h \in M} .
\end{aligned}
$$

From the construction, it follows that $c_{i}^{*} \in \mathfrak{C}^{S}$ for each $i \in N$. Define $\mathbf{e}_{3}=\left(S, \mathbf{c}^{*},(\bar{x}, \bar{y})\right)$ with $\mathbf{c}^{*}=\left(c_{i}^{*}\right)_{i \in N}$. Then, $\mathcal{C}\left(\mathbf{e}_{3}\right)=\mathcal{C}$. Next, for each $i \in N$, let us define $\widehat{c}_{i}: \mathbb{R}_{+}^{s} \rightarrow \mathbb{R}_{+}^{k}$ as: $\widehat{c}_{i}\left(x_{i}, y\right)=c_{i}^{m}\left(x_{i}\right)$ for any $y \in \mathbb{R}_{+}^{l}$. Define $\widehat{\mathbf{e}}_{1}=(S, \widehat{\mathbf{c}},(\bar{x}, \bar{y}))$ with $\widehat{\mathbf{c}}=\left(\widehat{c}_{i}\right)_{i \in N}$. Then, $\mathcal{C}\left(\widehat{\mathbf{e}}_{1}\right)=\mathcal{C}$. 
Compare $\mathbf{e}_{3}$ and $\widehat{\mathbf{e}}_{1}$. By construction, for any $(x, y) \in \mathbb{R}_{+}^{s}, \widehat{c}_{i}(x, y) \supseteq$ $c_{i}^{*}(x, y)$ holds for each $i \in N$. Moreover, for any $(x, y) \in \mathbb{R}_{+}^{s}$ with $(x, y) \leq$ $(\bar{x}, \bar{y})$, there exists $(\delta(x), \delta(y)) \in \mathbb{R}_{+}^{s}$ with $(\delta(x), \delta(y)) \geq(x, y)$ such that $\widehat{c}_{i}(x, y)=c_{i}^{*}(\delta(x), \delta(y))$. In fact, for instance, if $\delta(x)=x$ and $\delta(y)=$ $\left(y \vee \tau_{i}(x)\right)$, where $x \vee x^{\prime} \equiv\left(\max \left\{x_{h}, x_{h}^{\prime}\right\}\right)_{h \in M}$, then $\widehat{c}_{i}(x, y)=c_{i}^{*}(\delta(x), \delta(y))$ holds. Then, let us define a new commodity space $\mathbb{R}_{+}^{s} \times \mathbb{R}_{+}^{t(i)}$, with $t(i)=s$ and $\bar{w}^{i} \in \mathbb{R}_{+}^{t(i)} \backslash\{0\}$, and a new correspondence $\widehat{c}_{i}^{*}: \mathbb{R}_{+}^{s} \times \mathbb{R}_{+}^{t(i)} \rightarrow \mathbb{R}_{+}^{k}$ as: for any $(x, y) \in \mathbb{R}_{+}^{s}$,

(i) $\widehat{c}_{i}^{*}\left(x, y, \bar{w}^{i}\right)=\widehat{c}_{i}(x, y)$;

(ii) $\widehat{c}_{i}^{*}(x, y, 0)=c_{i}^{*}(x, y)$;

(iii) for any $w \leq \bar{w}^{i}$, there exists $\left(x^{\prime}, y^{\prime}\right) \in \mathbb{R}_{+}^{s}$ with $x \leq x^{\prime} \leq \delta(x)$ and $y \leq y^{\prime} \leq \delta(y)$ such that $\widehat{c}_{i}^{*}(x, y, w)=c_{i}^{*}\left(x^{\prime}, y^{\prime}\right)$; and

(iv) for any $w, w^{\prime} \leq \bar{w}^{i}$ with $w \geq w^{\prime}, \widehat{c}_{i}^{*}(x, y, w) \supseteq \widehat{c}_{i}^{*}\left(x, y, w^{\prime}\right)$.

(v) for any $w$ with $w \not \leq \bar{w}^{i}, \widehat{c}_{i}^{*}(x, y, w)=\widehat{c}_{i}^{*}\left(x, y, w \wedge \bar{w}^{i}\right)$.

Let us denote the set of commodities in $\mathbb{R}_{+}^{t(i)}$ by $T(i)$. Such $\widehat{c}_{i}^{*}$ can be constructed to meet $\widehat{c}_{i}^{*} \in \mathfrak{C}^{S \cup T(i)}$.

Given $\left(\widehat{c}_{i}^{*}\right)_{i \in N}$, let us define a new commodity space $\mathbb{R}_{+}^{s} \times\left(\prod_{i \in N} \mathbb{R}_{+}^{t(i)}\right)$ with $\left(\bar{w}^{i}\right)_{i \in N} \in \prod_{i \in N} \mathbb{R}_{+}^{t(i)}$, and a profile of new correspondences $\left(\widehat{c}_{i}^{* *}\right)_{i \in N}$ as follows: for each $i \in N, \widehat{c}_{i}^{* *}: \mathbb{R}_{+}^{s} \times\left(\prod_{i \in N} \mathbb{R}_{+}^{t(i)}\right) \rightarrow \mathbb{R}_{+}^{k}$ is given by: for each $\left(x, y, w_{1}, \ldots, w_{n}\right) \in \mathbb{R}_{+}^{s} \times\left(\prod_{i \in N} \mathbb{R}_{+}^{t(i)}\right), \widehat{c}_{i}^{* *}\left(x, y, w_{1}, \ldots, w_{n}\right)=\widehat{c}_{i}^{*}\left(x, y, w_{i}\right)$. Let us denote the set of commodities in $\prod_{i \in N} \mathbb{R}_{+}^{t(i)}$ by $T$. Define a new economy $\widehat{\mathbf{e}}^{* *} \equiv\left(S \cup T, \widehat{\mathbf{c}}^{* *},\left(\bar{x}, \bar{y},\left(\bar{w}^{i}\right)_{i \in N}\right)\right) \in \mathcal{E}$ with $\widehat{\mathbf{c}}^{* *} \equiv\left(\widehat{c}_{i}^{* *}\right)_{i \in N}$. Then, $\mathcal{C}\left(\widehat{\mathbf{e}}^{* *}\right)=\mathcal{C}$ still holds, since $\mathcal{C}\left(\widehat{\mathbf{e}}^{* *}\right)=\mathcal{C}\left(\mathbf{e}_{3}\right)=\mathcal{C}\left(\widehat{\mathbf{e}}_{1}\right)$.

Let $(\widehat{\mathbf{x}}, \widehat{\mathbf{y}}, \widehat{\mathbf{w}}) \in \varphi\left(\widehat{\mathbf{e}}^{* *}\right)$. By (WEE), we can assume that each $i \in N$ receives $\left(\widehat{x}_{i}, \widehat{y}_{i},\left(\bar{w}^{i}, \mathbf{0}_{-i}\right)\right)$ in the allocation $(\widehat{\mathbf{x}}, \widehat{\mathbf{y}}, \widehat{\mathbf{w}})$, since, by the construction of $\widehat{\mathbf{c}}^{* *}$, every commodity in $T(i)$ is only useful for $i \in N$ in the sense that this is useless for any other individual at $(\widehat{\mathbf{x}}, \widehat{\mathbf{y}}, \widehat{\mathbf{w}})$. Moreover, by (WEE) and (F), we can assume that $\widehat{c}_{i}^{* *}\left(\widehat{x}_{i}, \widehat{y}_{i},\left(\bar{w}^{i}, \mathbf{0}_{-i}\right)\right)=\widehat{c}_{i}^{* *}\left(\widehat{x}_{i}, \widehat{y}_{i}, \mathbf{0}\right)=c_{i}^{*}\left(\widehat{x}_{i}, \widehat{y}_{i}\right)$ for each $i \in N$. In fact, if $\widehat{c}_{i}^{* *}\left(\widehat{x}_{i}, \widehat{y}_{i},\left(\bar{w}^{i}, \mathbf{0}_{-i}\right)\right) \supsetneq \widehat{c}_{i}^{* *}\left(\widehat{x}_{i}, \widehat{y}_{i}, \mathbf{0}\right)$ for some $i \in N$, it implies that either $\sum_{i \in N} \widehat{y}_{i}<\bar{y}$ or $\widehat{c}_{j}^{* *}\left(\widehat{x}_{j}, \widehat{y}_{j},\left(\bar{w}^{j}, \mathbf{0}_{-j}\right)\right)=\widehat{c}_{j}^{* *}\left(\widehat{x}_{j}, \widehat{y}_{j}, \mathbf{0}\right)=$ $c_{j}^{*}\left(\widehat{x}_{j}, y_{j}\left(\widehat{x}_{j}\right)\right)$ with $\widehat{y}_{j}>y_{j}\left(\widehat{x}_{j}\right)$ holding for some other $j \in N$. Then, by reallocating $\bar{y}$ from $\left(\widehat{y}_{i}\right)_{i \in N}$ to $\left(y_{i}\left(\widehat{x}_{i}\right)\right)_{i \in N}$ with $\sum_{i \in N} y_{i}\left(\widehat{x}_{i}\right)=\bar{y}$, we can have $\widehat{c}_{i}^{* *}\left(\widehat{x}_{i}, y_{i}\left(\widehat{x}_{i}\right),\left(\bar{w}^{i}, \mathbf{0}_{-i}\right)\right)=\widehat{c}_{i}^{* *}\left(\widehat{x}_{i}, \widehat{y}_{i},\left(\bar{w}^{i}, \mathbf{0}_{-i}\right)\right)=\widehat{c}_{i}^{* *}\left(\widehat{x}_{i}, y_{i}\left(\widehat{x}_{i}\right), \mathbf{0}\right)$ for each $i \in N$. Thus, $\left(\widehat{x}_{i}, \widehat{y}_{i},\left(\bar{w}^{i}, \mathbf{0}_{-i}\right)\right)_{i \in N} \in \varphi\left(\widehat{\mathbf{e}}^{* *}\right)$ implies $\left(\widehat{x}_{i}, \widehat{y}_{i}, \mathbf{0}\right)_{i \in N} \in \varphi\left(\widehat{\mathbf{e}}^{* *}\right)$ by (WEE) and (F), and each of the commodities $T$ is useless for each $i \in N$ 
at $\left(\widehat{x}_{i}, \widehat{y}_{i}, \mathbf{0}\right)$.

Compare $\widehat{\mathbf{e}}^{* *}$ and $\widehat{\mathbf{e}}_{1}$. For any $(\mathbf{x}, \mathbf{y}) \in W E\left(\widehat{\mathbf{e}}_{1}\right),\left(\mathbf{x}, \mathbf{y},\left(\bar{w}^{i}, \mathbf{0}_{-i}\right)_{i \in N}\right) \in$ $W E\left(\widehat{\mathbf{e}}^{* *}\right)$ holds, and $\widehat{c}_{i}^{* *}\left(x_{i}, y_{i},\left(\bar{w}^{i}, \mathbf{0}_{-i}\right)\right)=\widehat{c}_{i}\left(x_{i}, y_{i}\right)$ holds for every $i \in N$. Then, by (INC), $(\widehat{\mathbf{x}}, \widehat{\mathbf{y}}) \in \varphi\left(\widehat{\mathbf{e}}_{1}\right)$. By $(\mathrm{F}), \mathcal{C}\left(\widehat{\mathbf{e}}^{* *}\right)=\mathcal{C}\left(\widehat{\mathbf{e}}_{1}\right)$ implies $\mathbf{c}\left(\varphi\left(\widehat{\mathbf{e}}^{* *}\right)\right)=$ $\mathbf{c}\left(\varphi\left(\widehat{\mathbf{e}}_{1}\right)\right)$.

Compare $\widehat{\mathbf{e}}^{* *}$ and $\mathbf{e}_{3}$. For any $(\mathbf{x}, \mathbf{y}) \in W E\left(\mathbf{e}_{3}\right),(\mathbf{x}, \mathbf{y}, \mathbf{0}) \in W E\left(\widehat{\mathbf{e}}^{* *}\right)$ holds, and $\widehat{c}_{i}^{* *}\left(x_{i}, y_{i}, \mathbf{0}\right)=c_{i}^{*}\left(x_{i}, y_{i}\right)$ holds for every $i \in N$. Then, by (INC), $(\widehat{\mathbf{x}}, \widehat{\mathbf{y}}) \in \varphi\left(\mathbf{e}_{3}\right)$. By $(\mathrm{F}), \mathcal{C}\left(\widehat{\mathbf{e}}^{* *}\right)=\mathcal{C}\left(\widehat{\mathbf{e}}_{3}\right)$ implies $\mathbf{c}\left(\varphi\left(\widehat{\mathbf{e}}^{* *}\right)\right)=\mathbf{c}\left(\varphi\left(\widehat{\mathbf{e}}_{3}\right)\right)$.

Compare $\widehat{\mathbf{e}}_{1}$ and $\mathbf{e}_{1}$. For any $\mathbf{x} \in W E\left(\mathbf{e}_{1}\right),(\mathbf{x}, \mathbf{y}) \in W E\left(\widehat{\mathbf{e}}_{1}\right)$ and $\widehat{c}_{i}\left(x_{i}, y_{i}\right)=c_{i}^{m}\left(x_{i}\right)$ hold for any $\mathbf{y} \in \mathbb{R}_{+}^{n l}$ and every $i \in N$. Moreover, any commodity in $L$ is useless for each $i \in N$ at $\left(x_{i}, \mathbf{0}\right)$ under $\widehat{\mathbf{e}}_{1}$. Thus, by (INC), $\widehat{\mathbf{x}} \in \varphi\left(\mathbf{e}_{1}\right)$. By $(\mathrm{F}), \mathcal{C}\left(\widehat{\mathbf{e}}_{1}\right)=\mathcal{C}\left(\mathbf{e}_{1}\right)$ implies $\mathbf{c}\left(\varphi\left(\widehat{\mathbf{e}}_{1}\right)\right)=\mathbf{c}\left(\varphi\left(\mathbf{e}_{1}\right)\right)$.

Note that $\mathbf{c}^{m}(\widehat{\mathbf{x}})=\mathbf{c}^{*}(\widehat{\mathbf{x}}, \widehat{\mathbf{y}})$. By $(\mathrm{F}), \mathcal{C}\left(\mathbf{e}_{3}\right)=\mathcal{C}\left(\mathbf{e}_{1}\right)$ implies $\mathbf{c}\left(\varphi\left(\mathbf{e}_{1}\right)\right)=$ $\mathbf{c}\left(\varphi\left(\mathbf{e}_{3}\right)\right)$.

Lemma 2: (WEE), (EII), and (INC) imply (SEII).

Proof. Let $\mathbf{e}_{1}, \mathbf{e}_{2} \in \mathcal{E}$ be such that $\mathbf{e}_{1}=\left(M, \mathbf{c}^{m}, \bar{x}\right) \in \mathcal{E}^{M}, \mathbf{e}_{2}=\left(L, \mathbf{c}^{l}, \bar{y}\right) \in$ $\mathcal{E}^{L}$, and $\mathcal{C}\left(\mathbf{e}_{1}\right)=\mathcal{C}\left(\mathbf{e}_{2}\right)$. Noting that (EII) implies $(\mathrm{F})$, by Lemma $\mathbf{1}$, $\mathbf{c}\left(\varphi\left(\mathbf{e}_{1}\right)\right)=\mathbf{c}\left(\varphi\left(\mathbf{e}_{2}\right)\right)$. Then, it follows that (EII) implies (SEII).

Lemma 3: (WEE), (F), (ECI), and (INC) imply (CI).

Proof. Let $\mathcal{C}, \mathcal{C}^{\prime} \in \Sigma$ be such that $\mathcal{C} \supseteq \mathcal{C}^{\prime}$ and $F(\mathcal{C}) \cap \mathcal{C}^{\prime} \neq \varnothing$. Then, there exist $\mathbf{e}_{1}=\left(M, \mathbf{c}^{m}, \bar{x}\right), \mathbf{e}_{2}=\left(L, \mathbf{c}^{l}, \bar{y}\right) \in \mathcal{E}$ such that $\mathcal{C}\left(\mathbf{e}_{1}\right)=\mathcal{C}$ and $\mathcal{C}\left(\mathbf{e}_{2}\right)=\mathcal{C}^{\prime}$. We will construct two new economies $\mathbf{e}_{3}=\left(S, \mathbf{c}^{k}, \bar{z}\right), \mathbf{e}_{4}=\left(S, \mathbf{c}^{k}, \bar{z}^{\prime}\right) \in \mathcal{E}$ such that $\bar{z} \geq \bar{z}^{\prime}, \mathcal{C}\left(\mathbf{e}_{3}\right)=\mathcal{C}$, and $\mathcal{C}\left(\mathbf{e}_{4}\right)=\mathcal{C}^{\prime}$. Then, (WEE), (F), (ECI), and $(\mathrm{INC})$ imply that $F\left(\mathcal{C}^{\prime}\right)=F(\mathcal{C}) \cap \mathcal{C}^{\prime}$.

Let $S$ be a set of commodities such that $\# S=s \equiv m+l$. Because $\mathcal{C}\left(\mathbf{e}_{1}\right) \supseteq \mathcal{C}\left(\mathbf{e}_{2}\right)$, for each $\mathbf{C} \in \mathcal{C}\left(\mathbf{e}_{2}\right)$, there exist $\mathbf{x} \in A\left(\mathbf{e}_{1}\right)$ and $\mathbf{y} \in A\left(\mathbf{e}_{2}\right)$ such that $c_{i}^{m}\left(x_{i}\right)=C_{i}=c_{i}^{l}\left(y_{i}\right)$ for each $i \in N$. Then, let us construct $\mathbf{c}^{*}=\left(c_{i}^{*}\right)_{i \in N}$ as in the proof of Lemma 1. Define $\mathbf{e}^{*}=\left(S, \mathbf{c}^{*},(\bar{x}, \bar{y})\right)$ with $\mathbf{c}^{*}=\left(c_{i}^{*}\right)_{i \in N}$. Then, $\mathcal{C}\left(\mathbf{e}^{*}\right)=\mathcal{C}\left(\mathbf{e}_{2}\right)$ holds. Define $\widehat{\mathbf{e}}_{1}=(S, \widehat{\mathbf{c}},(\bar{x}, \bar{y}))$ with $\widehat{\mathbf{c}}=\left(\widehat{c}_{i}\right)_{i \in N}$, as in the proof of Lemma 1. Then, $\mathcal{C}\left(\widehat{\mathbf{e}}_{1}\right)=\mathcal{C}\left(\mathbf{e}_{1}\right)$.

Next, define $\widehat{\mathbf{e}}^{* *} \equiv\left(S \cup T, \widehat{\mathbf{c}}^{* *},\left(\bar{x}, \bar{y},\left(\bar{w}^{i}\right)_{i \in N}\right)\right) \in \mathcal{E}$ with $\widehat{\mathbf{c}}^{* *} \equiv\left(\widehat{c}_{i}^{* *}\right)_{i \in N}$ exactly as in the proof of Lemma 1. Then, $\mathcal{C}\left(\widehat{\mathbf{e}}^{* *}\right)=\mathcal{C}\left(\mathbf{e}_{1}\right)$ holds. Moreover, define another new economy $\mathbf{e}^{* *} \equiv\left(S \cup T, \widehat{\mathbf{c}}^{* *},(\bar{x}, \bar{y}, \mathbf{0})\right) \in \mathcal{E}$ with $\widehat{\mathbf{c}}^{* *} \equiv$ $\left(\widehat{c}_{i}^{* *}\right)_{i \in N}$. Then, $\mathcal{C}\left(\mathbf{e}^{* *}\right)=\mathcal{C}\left(\mathbf{e}_{2}\right)$ holds. By $(\mathrm{ECI})$, we have $\mathbf{c}\left(\varphi\left(\mathbf{e}^{* *}\right)\right)=$ 
$\mathbf{c}\left(\varphi\left(\widehat{\mathbf{e}}^{* *}\right)\right) \cap \mathcal{C}\left(\mathbf{e}^{* *}\right)$. By (WEE), (F), (ECI), and (INC), and from Lemma 1, we obtain $\mathbf{c}\left(\varphi\left(\mathbf{e}^{* *}\right)\right)=\mathbf{c}\left(\varphi\left(\mathbf{e}_{2}\right)\right)$ and $\mathbf{c}\left(\varphi\left(\widehat{\mathbf{e}}^{* *}\right)\right)=\mathbf{c}\left(\varphi\left(\mathbf{e}_{1}\right)\right)$.

Lemma 4: (WEE), (F), (ECI), (ES), and (INC) imply (S).

Proof. Let $\mathcal{C}$ be a symmetric problem such that it contains identical assignments among which there is one, say $\mathbf{C}^{*}$, that is weakly efficient. Then, there exists $\mathbf{e}=\left(M, \mathbf{c}^{m}, \bar{x}\right) \in \mathcal{E}^{M}$ such that $\mathcal{C}(\mathbf{e})=\mathcal{C}$. In the following discussion, we will construct a new economy $\mathbf{e}^{\prime}=\left(L, \mathbf{c}^{l}, \bar{y}\right) \in \mathcal{E}^{L}$ such that $c_{i}^{l}=c_{j}^{l}$ for all $i, j \in N$ and $\mathcal{C}\left(\mathbf{e}^{\prime}\right) \supseteq \mathcal{C}$, and $G\left(\mathcal{C}\left(\mathbf{e}^{\prime}\right)\right)=G(\mathcal{C})$. Once such an economy is given, we will show that (WEE), (F), (ES), and (INC) imply $\mathbf{C}^{*} \in F\left(\mathcal{C}\left(\mathbf{e}^{\prime}\right)\right)$ and $\left\{G\left(\mathbf{C}^{*}\right)\right\}=G\left(F\left(\mathcal{C}\left(\mathbf{e}^{\prime}\right)\right)\right.$. Furthermore, since $\mathbf{C}^{*} \in \mathcal{C}$, by (WEE), $(\mathrm{F})$, (ECI), and (INC), $\mathbf{C}^{*} \in F(\mathcal{C})$ and $\left\{G\left(\mathbf{C}^{*}\right)\right\}=G(F(\mathcal{C})$ ) will follow from Lemma 3. This will imply that (S) holds.

Given $\mathbf{e}=\left(M, \mathbf{c}^{m}, \bar{x}\right) \in \mathcal{E}$ with $\mathcal{C}(\mathbf{e})=\mathcal{C}$, let us consider $\mathbf{e}_{\{1,2\}}=$ $\left(M,\left(c_{2}^{m}, c_{1}^{m}, \mathbf{c}_{-\{1,2\}}^{m}\right), \bar{x}\right) \in \mathcal{E}$. Then, since $\mathcal{C}(\mathbf{e})$ is symmetric, $\mathcal{C}\left(\mathbf{e}_{\{1,2\}}\right)$ is also symmetric, and $\mathcal{C}\left(\mathbf{e}_{\{1,2\}}\right)=\mathcal{C}$. Then, define $\mathbf{e}^{1,2}=\left(M^{1,2},\left(c_{i}^{2 m}\right)_{i \in N},(\bar{x}, \bar{x})\right)$ with $\# M^{1,2}=2 m$ as follows: for each $i \in\{1,2\}$, let, for each $\left(x_{1}, x_{2}\right) \in \mathbb{R}_{+}^{2 m}$, $c_{i}^{2 m}\left(x_{1}, x_{2}\right) \equiv c_{1}^{m}\left(x_{1}\right) \cap c_{2}^{m}\left(x_{2}\right)$. In addition, for any other $i \in N \backslash\{1,2\}$, let, for each $\left(x_{1}, x_{2}\right) \in \mathbb{R}_{+}^{2 m}, c_{i}^{2 m}\left(x_{1}, x_{2}\right)=c_{i}^{m}\left(x_{1}\right)$. Then, $\mathcal{C}\left(\mathbf{e}^{1,2}\right) \supseteq \mathcal{C}$ and $G\left(\mathcal{C}\left(\mathbf{e}^{1,2}\right)\right)=G(\mathcal{C})$. If $N=\{1,2\}$, then $\mathbf{e}^{1,2}$ is an economy with $c_{i}^{2 m}=c_{j}^{2 m}$ for all $i, j \in N$.

Given, $\mathbf{e}^{1,2} \in \mathcal{E}$, consider $\mathbf{e}_{\{1,3\}}^{1,2}=\left(M^{1,2},\left(c_{3}^{2 m}, c_{1}^{2 m}, c_{1}^{2 m}, \mathbf{c}_{-\{1,2,3\}}^{2 m}\right),(\bar{x}, \bar{x})\right)$ and $\mathbf{e}_{\{2,3\}}^{1,2}=\left(M^{1,2},\left(c_{1}^{2 m}, c_{3}^{2 m}, c_{1}^{2 m}, \mathbf{c}_{-\{1,2,3\}}^{2 m}\right),(\bar{x}, \bar{x})\right)$. Then, $\mathcal{C}\left(\mathbf{e}_{\{1,3\}}^{1,2}\right)=\mathcal{C}\left(\mathbf{e}_{\{2,3\}}^{1,2}\right)=$ $\mathcal{C}\left(\mathbf{e}^{1,2}\right)$. Define $\mathbf{e}^{1,2,3}=\left(M^{1,2,3},\left(c_{i}^{4 m}\right)_{i \in N},(\bar{x}, \bar{x}, \bar{x}, \bar{x})\right)$ with $\# M^{1,2,3}=4 m$ as follows: for each $i \in\{1,2,3\}$, let, for each $\left(x_{1}, x_{2}, x_{3}, x_{4}\right) \in \mathbb{R}_{+}^{4 m}, c_{i}^{4 m}\left(x_{1}, x_{2}, x_{3}, x_{4}\right)=$ $c_{1}^{2 m}\left(x_{1}, x_{2}\right) \cap c_{3}^{2 m}\left(x_{3}, x_{4}\right)$. In addition, for any other $i \in N \backslash\{1,2,3\}$, let, for each $\left(x_{1}, x_{2}, x_{3}, x_{4}\right) \in \mathbb{R}_{+}^{4 m}, c_{i}^{4 m}\left(x_{1}, x_{2}, x_{3}, x_{4}\right)=c_{i}^{2 m}\left(x_{1}, x_{2}\right)$. Then, $\mathcal{C}\left(\mathbf{e}^{1,2,3}\right) \supseteq \mathcal{C}\left(\mathbf{e}^{1,2}\right)$ and $G\left(\mathcal{C}\left(\mathbf{e}^{1,2,3}\right)\right)=G\left(\mathcal{C}\left(\mathbf{e}^{1,2}\right)\right)$. If $N=\{1,2,3\}$, then $\mathbf{e}^{1,2,3}$ is an economy with $c_{i}^{4 m}=c_{j}^{4 m}$ for all $i, j \in N$.

By repeating this procedure up to $n$, we obtain

$$
\mathbf{e}^{(1, \ldots, n)}=\left(M^{1, \ldots, n},\left(c_{i}^{2^{n-1} m}\right)_{i \in N},(\bar{x}, \ldots, \bar{x})\right)
$$

with $(\bar{x}, \ldots, \bar{x}) \in \mathbb{R}_{+}^{2^{n-1} m}$ and an identical profile $\left(c_{i}^{2^{n-1} m}\right)_{i \in N}$ such that $\mathcal{C}\left(\mathbf{e}^{(1, \ldots, n)}\right) \supseteq \mathcal{C}$ and $G\left(\mathcal{C}\left(\mathbf{e}^{(1, \ldots, n)}\right)\right)=G(\mathcal{C})$ hold. Then, since $\mathbf{C}^{*}$ is weakly efficient in $\mathcal{C}$, the identical assignment $\mathbf{C}^{*}$ is weakly efficient in $\mathcal{C}\left(\mathbf{e}^{(1, \ldots, n)}\right)$. 
Proof of Theorem 2. It is easy to check that $\varphi^{E}$ satisfies (WEE), (ES), (ECI), (EII), and (INC). Suppose that $\varphi$ satisfies (WEE), (ES), (ECI), (EII), and (INC). Then, by Lemmas 2, 3, and 4, $\varphi$ attains a bargaining solution which satisfies (WE), (S), (CI), and (II). By Theorem 1, $\varphi$ attains $F^{E}$.

In Theorem 2, the independence of the axioms can be checked as follows. For (WEE), the allocation rule $\varphi$ such that $\varphi(\mathbf{e})=\{\mathbf{0}\}$ for each $\mathbf{e}$ satisfies all of the axioms but (WEE); for (ES), the Nash allocation rule $\varphi^{N}$ which attains the Nash solution [Xu and Yoshihara (2006a)] to (opportunity)bargaining problems satisfies all of the axioms but (ES); for (ECI), the KalaiSmorodinsky allocation rule $\varphi^{K}$ which attains the Kalai-Smorodinsky solution [Xu and Yoshihara (2006a)] to (opportunity)-bargaining problems satisfies all of the axioms but (ECI); for (EII), an allocation rule which attains a proper subsolution of the egalitarian solution $F^{E}$ satisfies all of the axioms but (EII); and finally, for (INC), let us consider the following rule $\varphi^{*}$ :

$$
\varphi^{*}(\mathbf{e})= \begin{cases}\varphi^{E}(\mathbf{e}) & \text { if } c_{i}^{m}=c_{j}^{m} \text { for all } i, j \in N \text { in } \mathbf{e}=\left(M, \mathbf{c}^{m}, \bar{x}\right) \in \mathcal{E} \\ \varphi^{N}(\mathbf{e}) & \text { otherwise }\end{cases}
$$

This $\varphi^{*}$ satisfies (WEE), (ES), (ECI), and (EII), but (INC). This is because $\mathbf{c}\left(\varphi^{*}\left(\mathbf{e}_{1}\right)\right) \neq \mathbf{c}\left(\varphi^{*}\left(\mathbf{e}_{2}\right)\right)$ generally holds for $\mathcal{C}\left(\mathbf{e}_{1}\right)=\mathcal{C}\left(\mathbf{e}_{2}\right)$ if $c_{i}^{m}=c_{j}^{m}$ for all $i, j \in N$ in $\mathbf{e}_{1}$ and $c_{i}^{l} \neq c_{j}^{l}$ for some $i, j \in N$ in $\mathbf{e}_{2}$. Then, by Lemma $\mathbf{1}, \varphi^{*}$ violates (INC).

\section{Discussion and Conclusion}

In this paper, we have extended the standard bargaining model to situations in which players are characterized by their opportunity sets rather than by their utilities ${ }^{7}$ and in which the fair arbitrator makes the recommendation with the guiding principle of equal opportunity for all players. In such a setting, we have formulated our problems in terms of bargaining problems among players on opportunity assignments, defined the egalitarian solution in our context and studied it axiomatically. Most of the axioms used in our axiomatic characterization of the proposed solutions correspond to their counterparts in standard bargaining models, but formulated either in terms

\footnotetext{
${ }^{7}$ It may be remarked that, when $k=1$ (there is only one basic living condition), our model reduces to the standard non-convex bargaining problem.
} 
of opportunity assignments or directly in economic environments. We have discussed and commented on the axioms that are unique in our context.

In what follows, we shall present a discussion of our egalitarian solution and make a connection between our egalitarian solution and the notion of equality of opportunity proposed by Roemer (1998).

Structurally, the egalitarian solution of bargaining over opportunity assignments and the standard egalitarian solution to the problems of utility allocations are similar. However, their conceptual implications are quite different.

First, our $\varphi^{E}$ attempts to capture the idea of equality of opportunity, whereas the egalitarian solution for the standard bargaining problems is based on the idea of equality of outcome or equality of welfare. The egalitarian principle based on outcomes or welfares has been under critical scrutiny in recent years and rival theories of equality based on opportunities have been advocated by several people. For instance, Sen (1980) proposed equality of capabilities, which requires equalizing opportunities among individuals to realize their functionings, and contrasted and emphasized the difference between equality of outcome in any form and equality of capabilities in their respective performances. Our $\varphi^{E}$ can be viewed as a mechanism to implement the idea of equality of capabilities.

Secondly, as we discussed earlier in the Introduction and in Section 2, there are situations in which bargaining problems are about opportunity assignments rather than final outcomes or payoffs of players. In such cases, one plausible guiding principle is "leveling players' playing fields as much as possible" (Roemer (1998)). This guiding principle does not necessarily guarantee the equalization of players' final outcomes, and our egalitarian solution is a way to ensure that players have the same playing field. The egalitarian solution to the problems of bargaining over opportunity assignments seems to fit those situations well.

Thirdly, our $\varphi^{E}$ does not actually guarantee equal final outcomes for players. To see this, let us consider the situation discussed in Example 1. Given an allocation $\mathbf{x}$ recommended by $\varphi^{E}$ in an economy $\mathbf{e}=\left(M, \mathbf{c}^{m}, \bar{x}\right)$, each individual $i \in N$ is guaranteed to acquire any level of skills within $c_{i}^{m}\left(x_{i}\right)$ by devoting his effort appropriately. However, the exact levels of skills he actually acquires depend on his effort level. Suppose that every individual has the common utility function $u(\mathbf{b}, a)$, which is a function of realized skill vector $\mathbf{b}$ and the effort level $a$. Each individual $i$ chooses his effort level $a_{i}$ and $\mathbf{b}_{i}$ in order to maximize $u(\mathbf{b}, a)$ subject to $\mathbf{b} \in c_{i}^{m}\left(x_{i}\right)$. 
Let $v\left(c_{i}^{m}\left(x_{i}\right)\right) \equiv \max _{\mathbf{b} \in c_{i}^{m}\left(x_{i}\right), a} u(\mathbf{b}, a)$. The standard egalitarian solution recommends an allocation $\mathbf{x}^{\prime}$ in order to guarantee $v\left(c_{i}^{m}\left(x_{i}^{\prime}\right)\right)=v\left(c_{j}^{m}\left(x_{j}^{\prime}\right)\right)$ for any $i, j \in N$. On the other hand, $\varphi^{E}$ guarantees that, for a given ordering $R$ over opportunity sets, $\left(c_{i}^{m}\left(x_{i}\right), c_{j}^{m}\left(x_{j}\right)\right) \in I$ for any $i, j \in N$, but $\left[v\left(c_{i}^{m}\left(x_{i}\right)\right)=v\left(c_{j}^{m}\left(x_{j}\right)\right)\right]$ may not hold for some individuals $i, j \in N$. Therefore, the recommendation by $\varphi^{E}$ is much different from the recommendation of the standard egalitarian solution. In the context of Example 1, we believe that $\varphi^{E}$ is more plausible than the standard egalitarian solution. This is because the choices of $a_{i}$ and $\mathbf{b}_{i}$ are a matter of personal responsibility, and $\varphi^{E}$ delegates this personal responsibility to individual players while the standard egalitarian solution does not.

How is our approach in the paper related to the theory of equality of opportunity discussed by Roemer (1998)? There are some differences between our approach in this paper and Roemer's model (1998). For example, for Roemer (1998), the task is to propose a social welfare function that determines the optimal equal opportunity policy, while in this paper, we define and characterize "fair" solutions for bargaining problems based on players' opportunity sets. There are also similarities between our model and Roemer's model. In Roemer (1998), the resource allocation determined by the optimal policy is to guarantee any two individuals the equal opportunity of access to the same level of "advantages" regardless of their "types", if their effort rankings within their own "types" are identical. In our model, an individual's type in the sense of Roemer is reflected in the individual's opportunity correspondence, and a bargaining solution such as $F^{E}$ determines a resource allocation to guarantee an equitable assignment of opportunities among individuals, under which every individual may access to the same level of living-condition vectors.

\section{References}

1. Arneson, R. (1989): "Equality and equal opportunity for welfare," Philosophical Studies 56, 77-93.

2. Cohen, G.A. (1993): "Equality of what? On welfare, goods, and capabilities," in The Quality of Life, (edited by Nussbaum, M. and A. Sen), Oxford Univ. Press: Oxford, 9-29.

3. Gaertner, W. and Y. Xu (2006): "Capability as the Basis for a New 
Measure of the Standard of Living," Journal of Human Development 7(3), 311-321.

4. Gorman, W. (1980): "The demand for related goods: a possible procedure for analysing quality differentials in the egg market," Review of Economic Studies 47, 843-856.

5. Gotoh, R. and N. Yoshihara (2003): "A class of fair distribution rules a la Rawls and Sen," Economic Theory 22, 63-88.

6. Herrero, C. (1996): "Capabilities and utilities," Economic Design 2, 69-88.

7. Herrero, C. (1997): "Equitable opportunities: An extension," Economics Letters 55, 91-95.

8. Kalai, E., (1977): "Proportional Solutions to Bargaining Situations: Interpersonal Utility Comparisons," Econometirca 45, 1623-1630.

9. Kalai, E., and M. Smorodinsky (1975): "Other Solutions to Nash's Bargaining Problem," Econometirca 43, 513-518.

10. Kranich, L. (1996): "Equitable opportunities: An axiomatic approach," Journal of Economic Theory 71, 131-147.

11. Lancaster, K. J., (1966): "A new approach to consumer theory," Journal of Political Economy 74, 132-57.

12. Nash, J. F. (1950): "The bargaining problem," Econometrica, 18, 155162.

13. Nussbaum, M. (1988): "Nature, function and capability: Aristotle on political distribution," Oxford Studies in Ancient Philosophy, I (supplementary), 145-84.

14. Nussbaum, M. (1993): "Non-relative virtues: an Aristotelian approach", in M. Nussbaum and A.K. Sen (eds.), The Quality of Life, Clarendon Press, Oxford.

15. Nussbaum, M. (2000): Women and Human Development: The Capability Approach, Kali for Women, New Delhi. 
16. Pattanaik, P.K. and Y. Xu (1990): "On ranking opportunity sets in terms of freedom of choice," Recherches Economiques de Louvain, 56, 383-390.

17. Pattanaik, P.K. and Y. Xu (2000): "On ranking opportunity sets in economic environments," Journal of Economic Theory 93(1), 48-71.

18. Pattanaik, P.K. and Y. Xu (2007): "Minimal relativism, dominance, and standard of living comparisons based on functionings," Oxford Economic Papers 59(2), 354-374.

19. Roemer, J. E., (1988): "Axiomatic bargaining theory on economic environments," Journal of Economic Theory 45, 1-31.

20. Roemer, J.E. (1998): Equality of opportunity, Harvard Univ. Press: Cambridge.

21. Savaglio, E. and S. Vannucci (2006): "On the volume-ranking of opportunity sets in economic environments," mimeo.

22. Sen, A. K. (1980): "Equality of what?" in Tanner Lectures on Human Values. Vol. 1, (edited by McMurrin, S.), Cambridge Univ. Press: Cambridge.

23. Sen, A. K. (1985): Commodities and capabilities, Amsterdam: NorthHolland.

24. Sen, A. K. (2002): Rationality and freedom, Harvard University Press: Cambridge, MA.

25. Thomson, W. (1983): "Equity in exchange economies," Journal of Economic Theory 29, 217-244.

26. Xu, Y. (2002): "Functioning, capability, and the standard of living - an axiomatic approach," Economic Theory 20, 387-399.

27. Xu, Y. (2004): "On ranking linear budget sets in terms of freedom of choice," Social Choice and Welfare 22(1), 281-289.

28. $\mathrm{Xu}, \mathrm{Y}$. and N. Yoshihara (2006): "Alternative characterizations of three bargaining solutions for nonconvex problems," Games and Economic Behaviors 57, 86-92. 
29. $\mathrm{Xu}, \mathrm{Y}$. and N. Yoshihara (2006a): "Axiomatic bargaining theory on opportunity assignments," IER Discussion Paper N. 473, The Institute of Economic Research, Hitotsubashi University, Jan 2006.

30. Yoshihara, N., (2003): "Characterizations of bargaining solutions in production economies with unequal skills," Journal of Economic Theory 108, 256-285.

31. Yoshihara, N., (2006): "Solidarity and cooperative bargaining solutions," in A. Wieczorek, M. Malawski, and A. Wiszniewska-Matyszkiel, eds., Game Theory and Mathematical Economics, Banach Center Publications 70, Warszawa, pp. 317-330. 


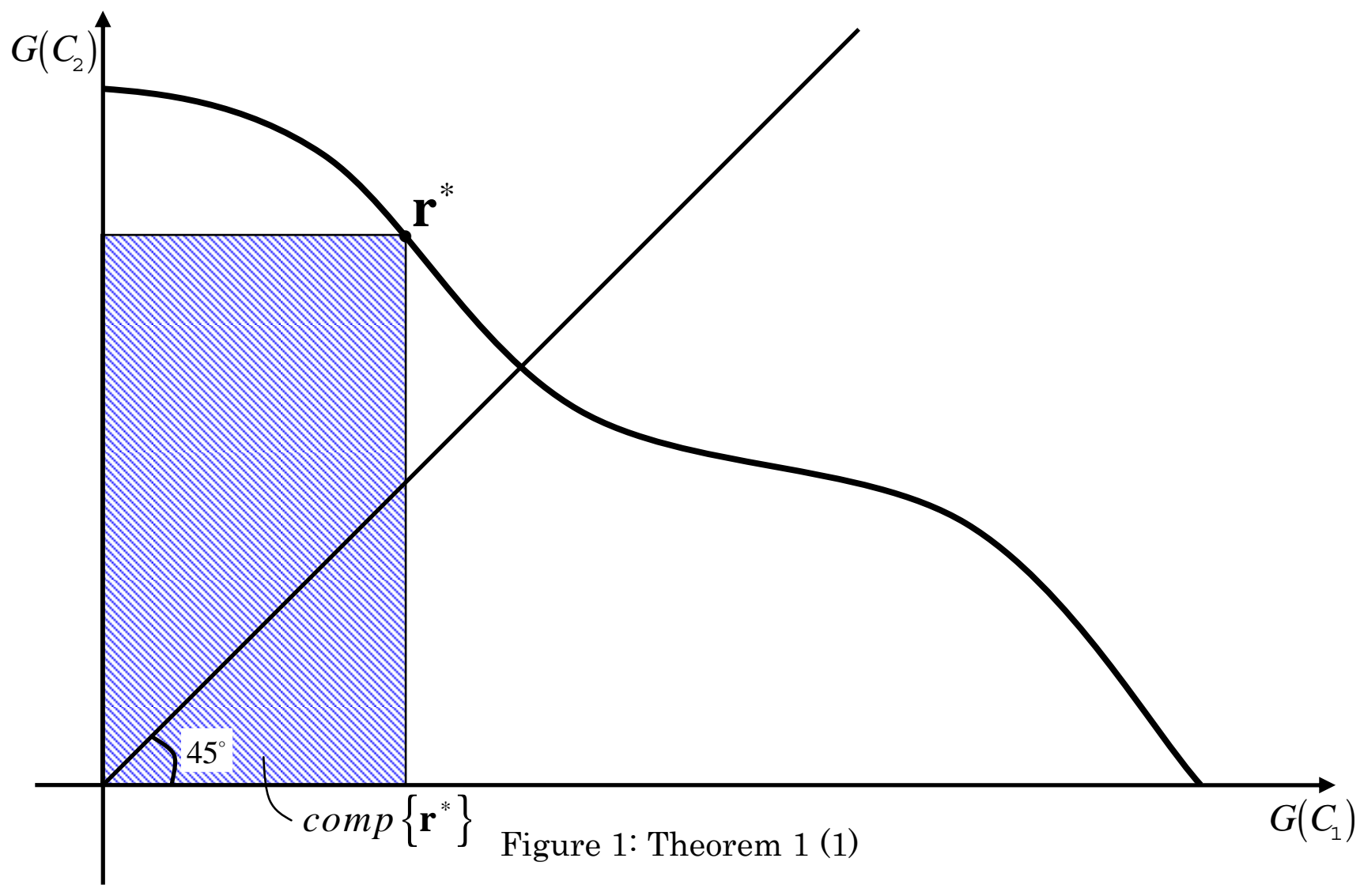




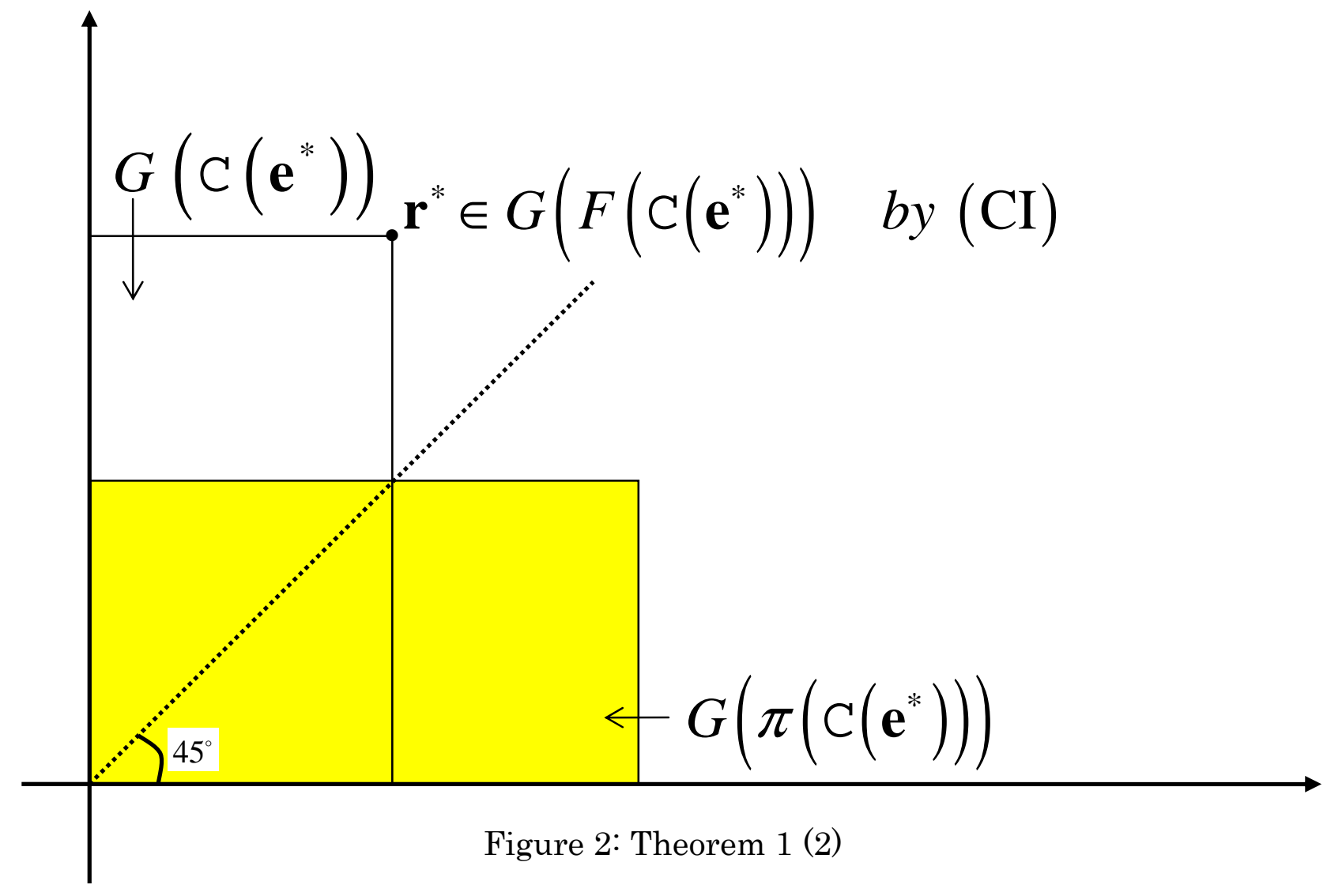




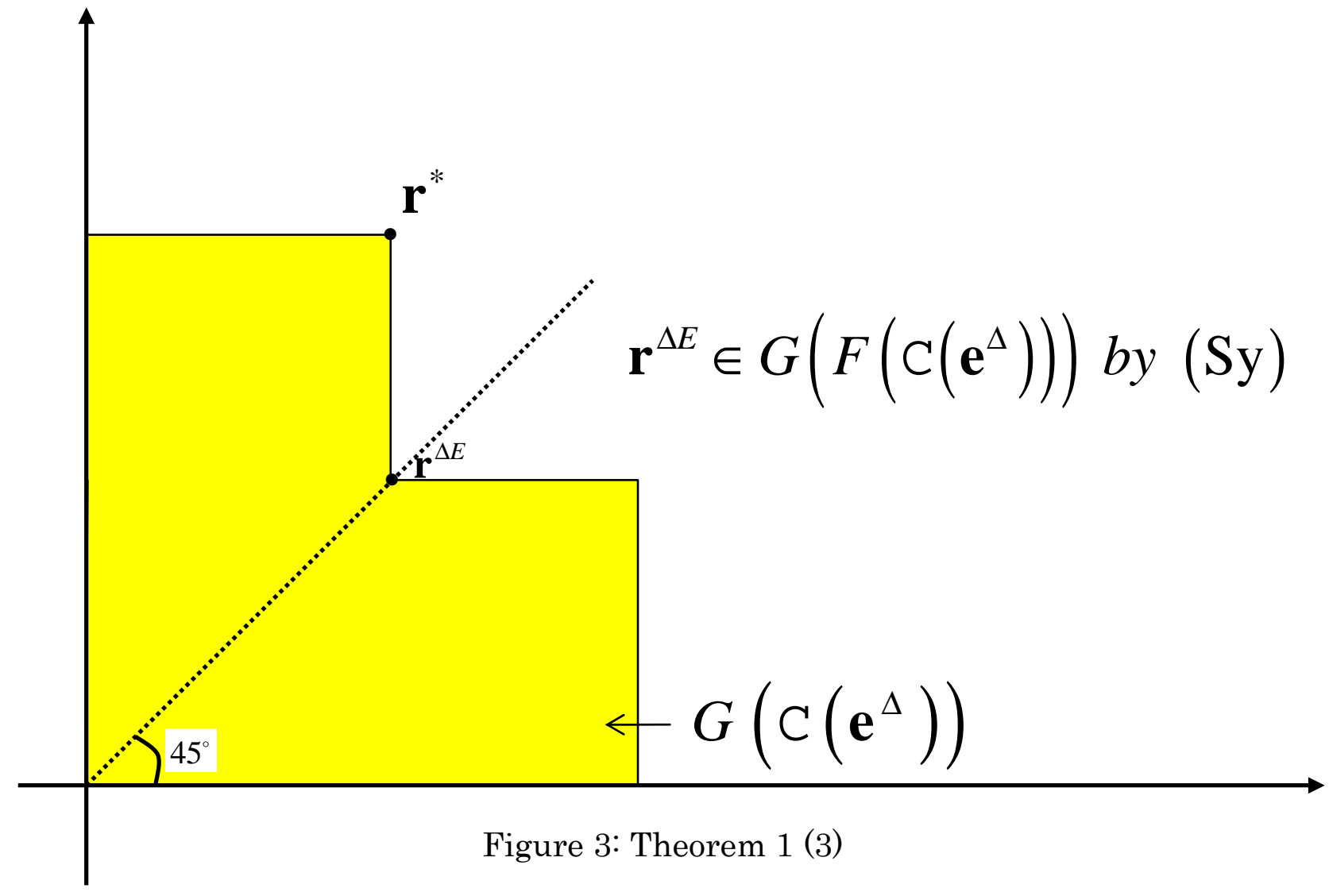




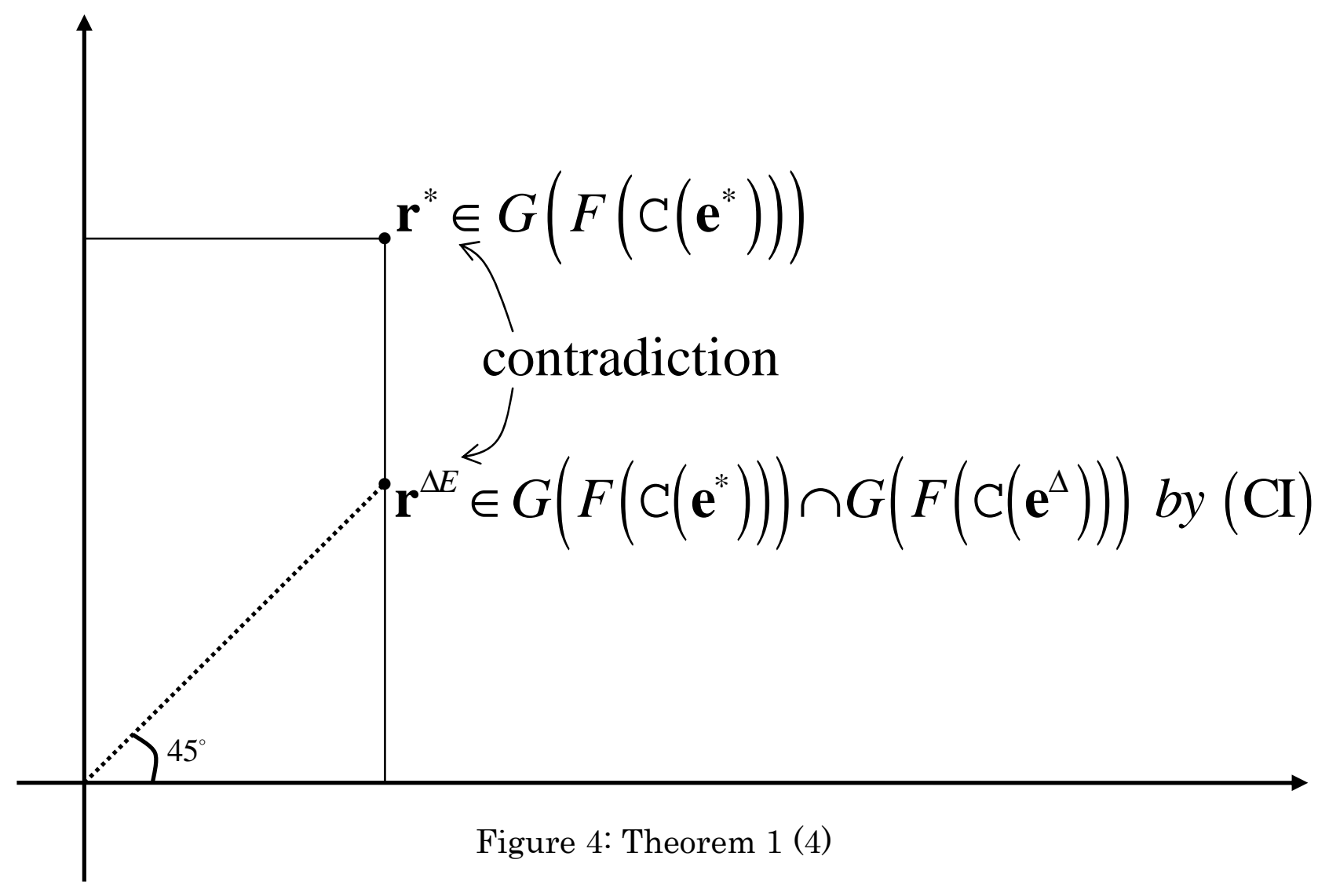

